\title{
La relación entre Inteligencia de Negocio e Inteligencia Competitiva: un análisis retrospectivo y bibliométrico de la literatura de 1959 a 2017
}

\author{
José Ricardo López-Robles*, José Ramón Otegi-Olaso*, Igone Porto-Gómez**, \\ Hamurabi Gamboa-Rosales***, Nadia Karina Gamboa-Rosales*** \\ *Universidad del País Vasco/Euskal Herriko Unibertsitatea, Departamento de Expresión Gráfica y Proyectos de Ingeniería, Bilbao \\ Correo-e: ricardolopezrobles@outlook.com | ORCID iD: http://orcid.org/0000-0003-3780-1955 \\ Correo-e: joserra.otegi@ehu.eus | ORCID iD: http://orcid.org/0000-0001-6023-8537 \\ **Universidad de Deusto, Deusto Business School, Bilbao \\ Correo-e: igone.porto@deusto.es | ORCID iD: http://orcid.org/0000-0003-2865-4818 \\ ***Universidad Autónoma de Zacatecas, Unidad Académica de Ingeniería Eléctrica, Zacatecas (México) \\ Correo-e: hamurabigr@uaz.edu.mx | ORCID iD: http://orcid.org/0000-0002-9498-6602 \\ Correo-e: ngamboa@conacyt.mx | ORCID iD: http://orcid.org/0000-0003-3421-8289
}

Recibido: 18-10-2018; 2a versión: 10-04-2019; Aceptado: 05-06-2019.

Cómo citar este artículo/Citation: López-Robles, J. R.; Otegi-Olaso, J. R.; Porto-Gómez, I.; Gamboa-Rosales, H.; GamboaRosales, N. K. (2020). La relación entre Inteligencia de Negocio e Inteligencia Competitiva: un análisis retrospectivo y bibliométrico de la literatura de 1959 a 2017. Revista Española de Documentación Científica, 43 (1), e256. https://doi.org/10.3989/ redc.2020.1.1619

Resumen: Actualmente, las organizaciones se encuentran inmersas en escenarios complejos y variables, pero sobre todo intensos en la producción, demanda y consumo de datos, información y conocimiento. Esto les ha llevado a desarrollar e incorporar modelos de inteligencia que mejoren su competitividad a través de la definición, recopilación, análisis, utilización y diseminación de información. No obstante, la complejidad de los escenarios y la diversidad de las organizaciones hace que no exista un modelo único de inteligencia, siendo la Inteligencia de Negocio e Inteligencia Competitiva los más difundidos y utilizados. En este sentido, este trabajo analiza los documentos disponibles en Scopus desde 1959 hasta 2017 sobre estos modelos, identificando las áreas temáticas más representativas, relaciones y evolución, estableciendo las bases para la integración de estos modelos bajo un mismo marco organizacional. Para ello, se ha evaluado su productividad y elaborado sus mapas científicos a través del uso de técnicas y herramientas bibliométricas.

Palabras clave: inteligencia de negocio; inteligencia competitiva; mapa científico; gestión de la información; análisis bibliométrico; redes de co-ocurrencia; modelos de inteligencia; inteligencia estratégica.

\section{The relationship between Business Intelligence and Competitive Intelligence: a retrospective analysis and literature review from 1959 to 2017}

Abstract: Nowadays, organizations are immersed in complex and variable but, above all, intense scenarios, in terms of production, demand and consumption of information. This, consequently, has led organizations to incorporate intelligence approaches into their strategy improving their competitiveness in an agile and flexible way through the definition, collection, analysis, exploitation and dissemination of information. Nevertheless, the complexity of scenarios and the diversity of the organizations themselves make it hard to have a unique intelligence model. This paper analyzes the documents hosted in Scopus from 1959 to 2017 about Business Intelligence and Competitive Intelligence -the most widespread intelligence approaches-, to identify the most representative thematic areas, their relationship, composition and evolution, with the aim of supporting researches and professionals on research, development with an hollistic intelligence approach. To this end, the performance of the main bibliometric indicators was evaluated and scientific maps of both intelligence approaches were produced.

Keywords: business intelligence; competitive intelligence; co-word analysis; conceptual evolution map; information management; science mapping analysis; intelligence models; strategic intelligence.

Copyright: (c) 2020 CSIC. Este es un artículo de acceso abierto distribuido bajo los términos de la licencia de uso y distribución Creative Commons Reconocimiento 4.0 Internacional (CC BY 4.0). 


\section{INTRODUCCIÓN}

Los desafíos tecnológicos, económicos y sociales en que viven actualmente las organizaciones han puesto de manifiesto que, para ser competitivas, deben pasar de una posición reactiva a una posición proactiva, con capacidad para innovar y adaptarse ágilmente a las singularidades que puedan suscitarse, haciendo uso inteligente de la información en todas sus áreas (Shujahat y otros, 2017; Eidizadeh y otros, 2017).

Para dar respuesta a estos desafíos, las organizaciones buscan desarrollar e implementar modelos de inteligencia que recopilen, analicen, interpreten y diseminen datos e información de alto valor añadido para su uso en la definición y ejecución de su estrategia, así como en los procesos de toma de decisiones (Carneiro, 2000).

Originalmente, el concepto de inteligencia ha estado ligado a los ámbitos militar, seguridad, político, económico y comercial. No obstante, el concepto ha ido evolucionando y especializándose en aspectos más organizacionales, dando lugar a distintos enfoques que mantienen dentro de sus objetivos recoger información sobre el entorno, almacenarla, analizarla, diseminarla y aplicarla, principalmente.

Desde el punto de vista organizacional, los enfoques más difundidos en las comunidades académica, científica y empresarial son los de Inteligencia de Negocio (Business Intelligence (BI)) e Inteligencia Competitiva (Competitive Intelligence (CI)) (López-Robles y otros, 2019).

Atendiendo a esta situación, este artículo tiene por objetivo establecer las bases para la integración de los enfoques de Inteligencia de Negocio e Inteligencia Competitiva bajo un mismo marco, identificando sus estructuras intelectuales, principales líneas de investigación, autores más productivos y publicaciones más citadas de cada enfoque de inteligencia, así como las tendencias a corto y medio plazo.

Para ello, se ha recurrido a la aplicación de la bibliometría, entendida como el conjunto de métodos y herramientas para evaluar y analizar un conjunto de publicaciones y sus citas con el fin de explorar su impacto en un campo especifico y cómo contribuyen al desarrollo del estado del arte en las principales áreas de investigación (Glenisson y otros, 2005). En concreto, se recurrió al análisis del desempeño y los mapas científicos, herramientas ampliamente aceptadas por las comunidades académica, científica y empresarial para analizar la literatura, siendo cada vez más valorados como una herramienta para medir la calidad y la productividad académica y científica (Moed y otros, 1995; Van Eck y Waltman, 2010; Leydesdorff y Rafols, 2009).
En último lugar, este artículo se estructura de la siguiente forma. En la Sección 2 se describe brevemente el estado del arte y cómo este trabajo pretende dar respuesta a la oportunidad de un modelo único de inteligencia. En la Sección 3, se expone la metodología implementada para evaluar el rendimiento de ambos enfoques, así como los parámetros de búsqueda utilizados para la creación de la base de datos utilizada. En la Sección 4, se exponen los resultados del análisis bibliométrico y las estructuras intelectuales de cada enfoque. Por último, en la Sección 5 se recogen las principales conclusiones del estudio.

\section{INTELIGENCIA DE NEGOCIO E INTELI- GENCIA COMPETITIVA}

Las organizaciones siempre han sido conscientes de la ventaja que representa disponer de la información adecuada en el momento oportuno, así como la desventaja que constituye la situación opuesta. Es por esto que en los últimos años las organizaciones han puesto especial atención en la oportunidad que representa la utilización inteligente de los datos, la información y el conocimiento en los procesos de toma de decisiones y su integración en la estrategia de las organizaciones (Salles, 2006; Martínez-Ávila y otros, 2014).

Uno de los primeros registros del concepto de inteligencia entendido como el uso inteligente de datos, información y conocimiento es la obra de Sun Tzu, El Arte de la guerra, que data del año 400 A.C. y que describe cómo en el ámbito militar la utilización de la información en el momento oportuno representa una ventaja clave para el éxito. En este sentido, la inteligencia ha seguido ligada durante mucho tiempo a los ámbitos militar, políticos, comerciales y de seguridad, siendo a mediados del siglo XX cuando despierta el interés de las organizaciones por aplicar este tipo de estrategias a sus actividades.

Este interés por parte de las organizaciones comienza por desarrollarse en los ámbitos de conocimiento de Administración y dirección de empresas, Marketing, Ciencias de la información, Ingeniería Informática, Prospectiva e Investigación de Operaciones (López-Robles y otros, 2018b; Schwarz, 2007; Sawyer y Huang, 2007; Fleisher y Blenkhorn, 2003).

Esto dio lugar a la utilización de distintos enfoques de inteligencia, que van desde perspectivas organizacionales y de mercado hasta configuraciones regionales o nacionales, siendo los más relevantes para las organizaciones por su aportación a la disciplina de inteligencia: Inteligencia de Negocio (Luhn, 1958; Gilad y Gilad, 1985), Inteligencia 
Competitiva (Gilad, 1989; Fuld, 1985), Inteligencia Empresarial (Cohen y Czepiec, 1988; Bullinger, 2005; Glynn, 1996), Inteligencia de Mercado (Capo, 1983; Kohli y otros, 1993) e Inteligencia Tecnológica (Porter y Detampel, 1995; Kostoff y Scaller, 2001; Lichtenthaler, 2003; Abrahams y otros, 2012), principalmente.

López-Robles y otros (2019) hacen una revisión detallada de los enfoques de inteligencia presentes en la literatura durante los últimos 30 años, analizando su evolución, relación y rendimiento, siendo la Inteligencia de Negocio, vinculada principalmente al ámbito de la informática, y la Inteligencia Competitiva, más cercana al mundo organizacional, los enfoques más desarrollados y difundidos dentro de la literatura (Liebowitz, 2006; López-Robles y otros, 2018a; Luhn, 1958; Prescott, 1992). No obstante, la revisión antes mencionada propone como futuras líneas de investigación el análisis de los enfoques de inteligencia de forma individual o conjunta en función de los objetivos.

En este sentido, el primero de estos enfoques se define generalmente como la habilidad de aprender de las relaciones de hechos presentados de forma que guíen las acciones hacia una meta, mientras que el segundo se describe como la selección, colección, interpretación y distribución de información pública de importancia estratégica para la toma de decisiones (Cleland y King, 1975; Zinkhan y Gelb, 1985; Gilad, 1989).

Asimismo, las comunidades académica, científica y empresarial han ido desarrollando de forma paralela estos enfoques, poniendo de manifiesto su coexistencia y complementariedad, así como la oportunidad que representa integrarlos bajo un mismo marco (Kochen, 1989; Walker, 1994; Desai y Bawden, 1993; López-Robles y otros, 2018c).

Este nuevo contexto impulsa la creación de modelos avanzados basados en el uso inteligente de los datos, la información y el conocimiento para la mejora de la competitividad de las organizaciones, incluyendo su capacidad para innovar a través del uso de las nuevas tecnologías (Von Krogh y otros, 2000; Rodríguez-Pallares, 2016). En consecuencia, para integrar ambos enfoques resulta necesario conocer sus estructuras intelectuales y la relación de éstas. Para ello, se han utilizado distintos métodos y herramientas bibliométricas que permiten evaluar y analizar las publicaciones relacionadas con estos enfoques y sus citas, con el fin último de explorar su impacto en el campo de la inteligencia y cómo contribuyen al desarrollo del estado del arte (Glenisson y otros, 2005; Moed y otros, 1995; Van Eck y Waltman, 2010; Leydesdorff y Rafols, 2009; González-Valiente, 2016).

\section{METODOLOGÍA}

Para analizar de qué forma se relacionan los enfoques de Inteligencia de Negocio e Inteligencia Competitiva y establecer un contexto común para el desarrollo de ambos, se decidió evaluar el rendimiento e impacto de cada enfoque a través del análisis de los principales indicadores bibliométricos de 1958 a 2017 (Glanzel, 2003; Rodriguez-Bolivar y otros, 2018; Moreno-Fernández y Moreno-Crespo, 2016; Arguimbau-Vivó y otros, 2013), y la confección de sus respectivos mapas científicos o mapas de coocurrencia en base a las palabras claves definidas por los autores en sus publicaciones, que serán reconocidas como líneas de investigación utilizando VOSviewer (Liu y otros, 2015; Cobo y otros, 2011). Para ello, se han realizado las siguientes acciones: (1) Recuperación y procesamiento de datos (Lagar Barbosa y otros, 2014; González-Valiente, 2016; Cabeza-Ramírez y otros, 2018), (2) Confección y normalización de los mapas científicos (Cantos-Mateos y otros, 2013), y (3) Visualización y análisis de las principales relaciones de los temas de investigación (Zhao y Strotmann, 2015; Cuartas y otros, 2014).

Tomando en cuenta lo anterior, se decidió desarrollar el análisis bibliométrico y los mapas científicos utilizando los documentos disponibles entre 1958 y 2017 en la base de datos Scopus. Dichos documentos y su información bibliográfica (año de publicación, título, autores, afiliación, país, revista o congreso, citas recibidas, entre otros) han sido recopilados en texto plano el 30 de julio de 2018, utilizando las siguientes consultas avanzadas:

- Consulta 1 (C1): TITLE-ABS-KEY ("Business Intelligence" OR "Competitive Intelligence") para recabar los documentos que hacen mención a cualquiera de los dos enfoques.

- Consulta 2 (C2): TITLE-ABS-KEY ("Business Intelligence") para conseguir los documentos que hacen mención únicamente al enfoque de Inteligencia de Negocio.

- Consulta 3 (C3): TITLE-ABS-KEY ("Competitive Intelligence") para obtener los documentos que hacen mención al enfoque de Inteligencia Competitiva.

Es importante mencionar que las consultas y los mapas científicos se han realizado utilizando los conceptos en inglés, atendiendo al hecho de que el $96 \%$ de los documentos se encuentran publicados en este idioma. No obstante, el resto de documentos presentan título, resumen y palabras clave también en inglés por lo que el análisis cubre la totalidad de la literatura existente. Además, las búsquedas han sido limitadas hasta el año 2017, atendiendo al hecho de contar con datos comparables por años completos. 
Asimismo, se examinaron manualmente todos los registros recuperados para garantizar su pertinencia y homogeneidad, a fin de evitar que un mismo documento, autor o cualquier otro elemento pudiera aparecer con dos o más variantes. En los casos en los que se registró alguna diferencia o falta de información, se examinó el documento completo para su clasificación.

Así mismo, se analizaron los principales indicadores de rendimiento bibliométrico, tanto de forma individual como conjunta, resaltando la productividad de los autores en términos de publicaciones y citas, países, áreas de conocimiento y fuentes de información. También, para establecer un nivel común de comparación entre los autores y las publicaciones se evaluaron los resultados aplicando el $h$-index y $H$-Classics (Alonso y otros, 2009; Schreiber, 2007; Schubert, 2007; Martínez y otros, 2014; Hirsch, 2005).

Por último, para la visualización de los mapas científicos se utilizó la herramienta bibliométrica VOSviewer, que agrupa las palabras clave incluidas por los autores para su identificación en la base de datos y a las que se denomina temas de investigación. Para este análisis, los temas de investigación tienen una co-ocurrencia mínima de 25 veces y una fuerza mínima de enlace de 50 relaciones entre ellos, lo que asegura un peso y representatividad dentro del desarrollo de la literatura. Además, se registran los 200 enlaces principales, asegurando la aparición de los temas de investigación más relevantes y con el mayor número de enlaces de cada enfoque de inteligencia, así como las conexiones entre estos, facilitando con ello la definición de una estructura conceptual conjunta y el análisis de su evolución (Callon y otros, 1991; He, 1999; Sternitzke y Bergmann, 2009; González-Valiente, 2016).

\section{RESULTADOS}

A continuación, se analiza el rendimiento de los enfoques de Inteligencia de Negocio e Inteligencia Competitiva, en términos de publicaciones, citas, distribución geográfica, áreas de investigación, fuentes de información y autores más referentes. Además, sus correspondientes mapas científicos, donde se establecen los temas de investigación más representativos, su relación, composición y evolución, utilizando VOSviewer.

\subsection{Publicaciones, citas, tipología de publica- ciones, distribución geográfica, áreas de in- vestigación y fuentes de información}

Tomando en cuenta las consultas avanzadas realizadas, la consulta $\mathrm{C} 1$ es la más extensa con un total de 10.316 documentos desde 1959 hasta 2017 (111 en acceso abierto), mientras que las con- sultas C2 y C3 recuperaron 6.031 (93 en acceso abierto) y 5.920 (42 acceso abierto) documentos, respectivamente. Asimismo, la consulta C2 presenta registros a partir de 1975, aunque dentro de la literatura empresarial se reconocen algunas publicaciones anteriores a dicha fecha.

En la Figura 1 se muestra la distribución anual de las publicaciones relacionadas con los enfoques de Inteligencia de Negocio e Inteligencia Competitiva desde 1959 hasta 2017.

En términos de producción, se pueden destacar cuatro hitos relacionados con el crecimiento de los enfoques de Inteligencia de Negocio e Inteligencia Competitiva en la literatura. El primer hito, refleja la baja productividad que se da desde 1959 hasta 1987, periodo en el cual solo se registraron 17 publicaciones ( 5 corresponden a Inteligencia de Negocio y 12 a Inteligencia Competitiva). El segundo hito se registra en 2007, cuando se alcanza el valor máximo de publicaciones relacionadas con ambos enfoques (1.040 publicaciones) y el valor máximo de publicaciones para Inteligencia Competitiva (856 publicaciones). El tercer hito, se registra en 2008 , cuando las publicaciones de Inteligencia Competitiva caen hasta las 313 publicaciones, propiciado por la disminución de eventos de especialización y el uso más intensivo de otros enfoques de inteligencia. Además, este mismo año, Inteligencia de Negocio sobrepasa por primera vez a Inteligencia Competitiva en términos de producción. Por último, el cuarto hito corresponde al registro del valor máximo de publicaciones relacionadas con Inteligencia de Negocio (587 publicaciones) en 2014.

En términos de tipología de documentos, la consulta $\mathrm{C} 1$ se compone principalmente de Publicaciones de congresos (4.562), Artículos (3.794) y Revisiones (799), mientras que la consulta C2 lo hace Publicaciones de congresos (3.159) y Artículos (1.904), y la consulta C3 por Publicaciones de congresos (2.539) y Artículos (2.292). En todos los casos se observa un equilibrio entre las Publicaciones de congresos y los Artículos, lo que denota una actividad de investigación y desarrollo constante y la realización de eventos especializados.

En la Figura 2 se muestra la distribución de las citas recibidas por las publicaciones asociadas a los enfoques de inteligencia según la base de datos Scopus al 30 de julio de 2018.

Al igual que en el caso de las publicaciones, la distribución de las citas muestra una tendencia positiva de 1959 a 2017. Durante este período, se registraron 78.111 citas para ambos enfoques de inteligencia. Un aspecto a resaltar es el hecho de que el enfoque de Inteligencia Competitiva ha mantenido un crecimiento constante, siendo el concepto más citado hasta 2017, cuando la Inteligencia de Negocio se convierte en el enfoque más citado. 
Figura 1. Distribución anual de publicaciones alojadas en Scopus de 1959-2017

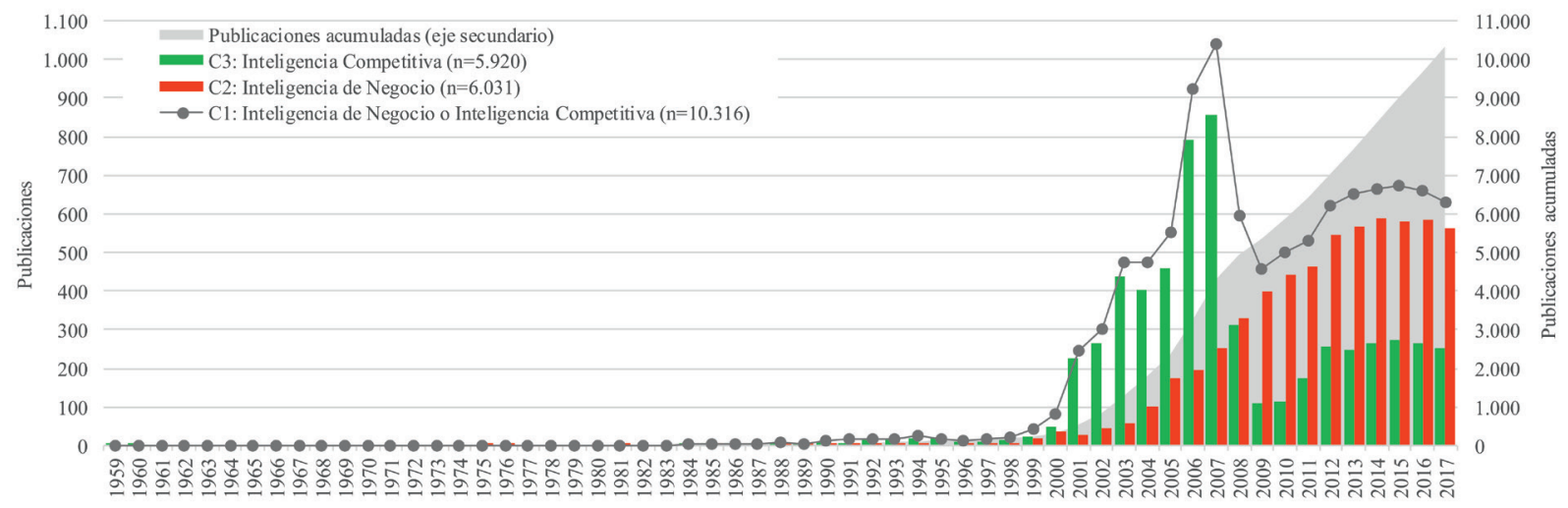

Figura 2. Distribución anual de citas alojadas en Scopus de 1959-2017

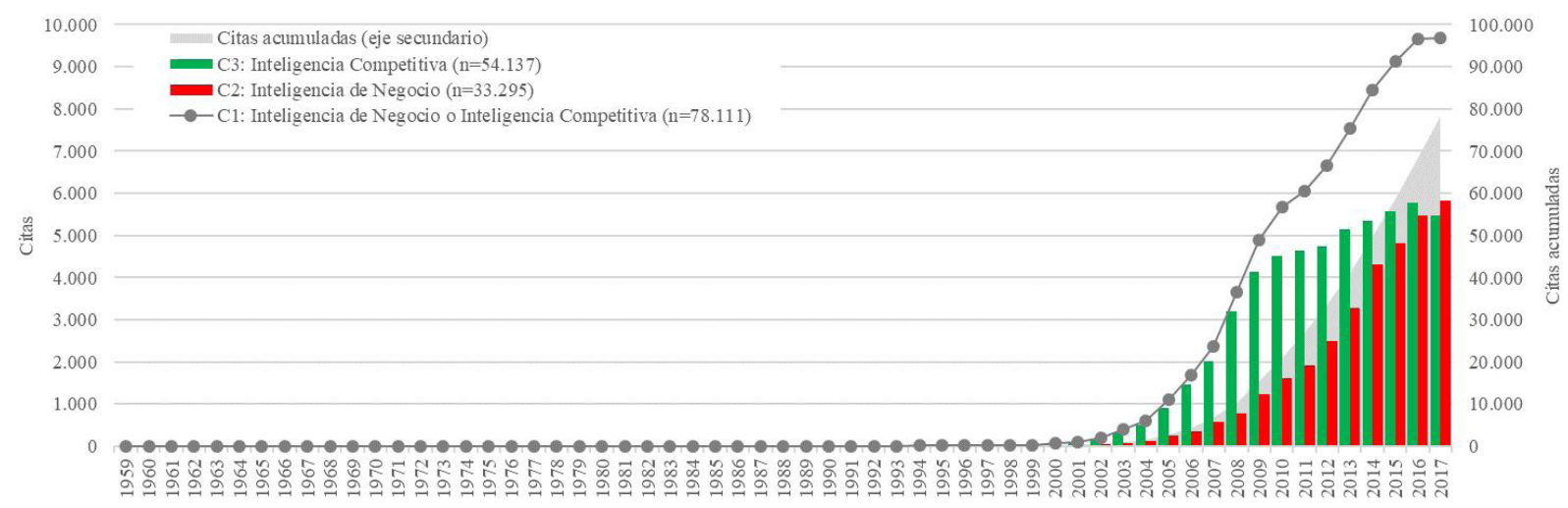

Por otro lado, los ámbitos de conocimiento cubiertos por los enfoques de Inteligencia de Negocio e Inteligencia Competitiva desde 1959 a 2017 no presentan apenas variación, siendo los más relevantes en conjunto: Computer Science (5.407), Engineering (3.422), Business, Management and Accounting (2.474) y Decision Sciences (1.452). Además, las tres principales fuentes de información para ambos enfoques son: Lecture Notes in Computer Science (incluyendo las subseries Lecture Notes in Artificial Intelligence y Lecture Notes in Bioinformatics) (434), Lecture Notes in Business Information Processing (154) y ACM International Conference Proceedings Series (112), tal como se puede observar en la Tabla I que describe las principales fuentes para el campo de inteligencia en el periodo definido.

Por último, los países más productivos tomando en cuenta ambos enfoques son: Estados Unidos (2101), China (977), Alemania (639), Reino Unido (510), Canadá (388), Australia (353), India (349), Francia (289), Italia (281) y España (253). Además, cabe mencionar que India (Inteligencia de Negocio), Italia (Inteligencia de Negocio) y España (Inteligencia Competitiva) son los únicos países identificados entre los más productivos que solo aparecen dentro de un único enfoque de inteligencia, lo que denota su especialización, tal como se puede observar en la Tabla II.

\subsection{Autores más productivos y autores más citados}

En la sección anterior, se ha comprobado que los enfoques de Inteligencia de Negocio e Inteligencia Competitiva presentan una actividad constante y en crecimiento, características claves a la hora de determinar la evolución y consolidación de las áreas de conocimiento que cubren. En este sentido, para complementar lo anterior, a continuación, se identifican quiénes son los autores más productivos y citados.

En la Tabla III se muestran los autores más productivos en el campo de la Inteligencia de Negocio e Inteligencia Competitiva entre 1959 y 2017. Dentro de los autores más productivos, destaca Yeoh, W., Baars, H., Mylopoulos, J., Golfarelli, M., Kemper, H.G., Rizzi, S., Machado, J. y Schrefl, M., que se encuentran presentes en ambos enfoques de inteligencia. 
Tabla I. Fuentes más productivas en el campo de la inteligencia de 1959 a 2017

\begin{tabular}{|l|c|c|c|c|}
\hline \multicolumn{1}{|c|}{ Revista (país) } & $\begin{array}{c}\text { SJR } \\
\mathbf{( 2 0 1 7 )}\end{array}$ & Mejor cuartil (ámbito) & $\begin{array}{c}\text { Publicaciones } \\
\text { (h-index) }\end{array}$ & $\begin{array}{c}\text { Citas totales } \\
\text { (auto citas) }\end{array}$ \\
\hline $\begin{array}{l}\text { Lecture Notes in Computer Science } \\
\text { (Alemania) }\end{array}$ & 0,295 & Q2 (Computer science) & $411.651(296)$ & $\begin{array}{c}781.750 \\
(229.086)\end{array}$ \\
\hline $\begin{array}{l}\text { Lecture Notes in Business } \\
\text { Information Processing (Alemania) }\end{array}$ & 0,222 & $\begin{array}{c}\text { Q3 (Business and } \\
\text { International Management) }\end{array}$ & $6,918(35)$ & $10.504(1.900)$ \\
\hline $\begin{array}{l}\text { ACM International Conference } \\
\text { Proceeding Series (Estados Unidos) }\end{array}$ & 0,159 & $\begin{array}{c}\text { Sin cuartil (Computer } \\
\text { science) }\end{array}$ & $39.975(83)$ & $41.506(2.960)$ \\
\hline $\begin{array}{l}\text { CEUR Workshop Proceedings } \\
\text { (Estados Unidos) }\end{array}$ & 0,167 & $\begin{array}{c}\text { Sin cuartil (Computer } \\
\text { science) }\end{array}$ & $33.516(35)$ & $20.500(4.195)$ \\
\hline $\begin{array}{l}\text { Communications in Computer and } \\
\text { Information Science (Alemania) }\end{array}$ & 0,170 & Q3 (Computer science) & $33.311(35)$ & $22.319(2.596)$ \\
\hline $\begin{array}{l}\text { Journal of Intelligence Studies In } \\
\text { Business (Suecia) }\end{array}$ & 0,293 & $\begin{array}{c}\text { Q2 (Business, Management } \\
\text { and Accounting) }\end{array}$ & $112(6)$ & $129(66)$ \\
\hline $\begin{array}{l}\text { IEEE International Engineering } \\
\text { Management Conference (China) }\end{array}$ & 0,104 & Sin cuartil (Enginnering) & $1.677(17)$ & $390(85)$ \\
\hline $\begin{array}{l}\text { Decision Support Systems } \\
\text { (Holanda) }\end{array}$ & 1,656 & $\begin{array}{c}\text { Q1 (Management } \\
\text { Information Systems) }\end{array}$ & $2.989(115)$ & $24.782(2.396)$ \\
\hline $\begin{array}{l}\text { Expert Systems with Applications } \\
\text { (Reino Unido) }\end{array}$ & 1,271 & Q1 (Engineering) & $11.234(145)$ & $110.187(14.442)$ \\
\hline DB2 Megazine (Estados Unidos) & 0,101 & Q4 (Information Systems) & $381(3)$ & $20(2)$ \\
\hline
\end{tabular}

Tabla II. Países más productivos en el campo de la inteligencia de 1959 a 2017

\begin{tabular}{|l|l|l|}
\hline $\begin{array}{l}\text { (C1) BI+CI (n=10.316) } \\
\text { (Publicaciones) País }\end{array}$ & $\begin{array}{l}\text { (C2) BI (n=6.031)(Publicaciones) } \\
\text { País }\end{array}$ & $\begin{array}{l}\text { (C3) CI (n=5.920)(Publicaciones) } \\
\text { País }\end{array}$ \\
\hline (2.101) Estados Unidos & $(1.128)$ Estados Unidos & $(1.280)$ Estados Unidos \\
\hline (977) China & (506) China & (596) China \\
\hline (639) Alemania & (477) Alemania & (324) Reino Unido \\
\hline (510) Reino Unido & (300) India & (294) Alemania \\
\hline (388) Canadá & $(274)$ Australia & (225) Canadá \\
\hline (353) Australia & $(255)$ Reino Unido & (170) Australia \\
\hline (349) India & $(237)$ Canadá & (154) Francia \\
\hline (289) Francia & $(231)$ Italia & (153) Taiwán \\
\hline (281) Italia & $(187)$ Portugal & $(147)$ España \\
\hline (253) España & $(180)$ Francia & $(133)$ Brasil \\
\hline
\end{tabular}

Tabla III. Autores más productivos en el campo de la inteligencia de 1959 a 2017

\begin{tabular}{|l|l|}
\hline $\begin{array}{l}\text { (C2) BI (n=6.031) } \\
\text { (Publicaciones) Autor(es) }\end{array}$ & $\begin{array}{l}\text { (C3) CI (n=5.920) } \\
\text { (Publicaciones) Autor(es) }\end{array}$ \\
\hline (34) Dayal, U. & (17) Rothberg, H.N \\
\hline (29) Yeoh, W. & (15) Trujillo, J. \\
\hline (25) Baars, H & (14) Maté, A. \\
\hline (24) Marjanovic, O.; Mylopoulos, J. & (13) Erickson, G.S.; Yeoh, W. \\
\hline (23) Golfarelli, M.; Rizzi, S.; Trujillo, J. & (12) Baars, H.; Calof, J.; James, D.; Rizzi, S.; Schrefl, M. \\
\hline (21) Bernardino, J.; Felden, C.; Pedersen, T.B.; Winter, R. & (11) Chung, W.; Mylopoulos, J.; Tinham, B. \\
\hline (19) Chung, W.; Kemper, H.G.; Maté, A.; Shi, Y. & (10) Abelha, A.; Golfarelli, M.; Machado, J. \\
\hline (18) Abelha, A.; Castellanos, M.; Popovič, A. & (9) Bartes, F.; Quoniam, L.; Søilen, K.S.; Wright, S.; Yu, E. \\
\hline (17) Arnott, D.; Machado, J. & $\begin{array}{l}\text { (8) Chase, L.; Chen, H.; Dinter, B.; Gonzales, M.L.; } \\
\text { Kemper, H.G.; Neumayr, B.; Portela, F.; Richards, G. }\end{array}$ \\
\hline (16) Santos, M.Y.; Schrefl, M. & $\begin{array}{l}\text { (7) Buonicore, A.J.; Chen, G.; Dou, H.; Goul, M.; } \\
\text { O'Donnell, P.; Prescott, J.E.; Romero, O.; Vogel, B. }\end{array}$ \\
\hline
\end{tabular}


En algunos puestos de la Tabla III se han registrado empates entre diferentes autores, por lo que se ha procedido a incluirlos en orden alfabético. Este criterio se aplica a todas las tablas incluidas en esta publicación.

En este sentido, Yeoh, W. (profesor de la Universidad de Deakin) destaca por ser uno de los investigadores más relevantes en el estudio del enfoque de Inteligencia de Negocios y técnicas de análisis. Baars, H. y Kemper, H.G. (profesores de la Universidad de Stuttgart) centran su actividad de investigación en la Inteligencia de Negocio y sus principales áreas de aplicación. Mylopoulos, J. (profesor en la Universidad de Toronto) por liderar proyectos de reingeniería de software, gestión del conocimiento y gestión de datos, áreas de conocimiento compartidas entre los dos enfoques de inteligencia analizados en esta publicación. Rizzi, S. (profesor de la Universidad de Bolonia) se especializa en ingeniería de software e Inteligencia de Negocio.

Golfarelli, M. (profesor asociado de la Universidad de Bolonia) desarrolla investigaciones en el ámbito de los sistemas de la información e Inteligencia de Negocio. Machado, J. (profesor en la Universidad del Minho) lidera proyectos de investigación en los ámbitos de los sistemas de información y Schrefl, M. (profesor de la Universidad de Linz) desarrolla proyectos de Inteligencia de Negocios, sistemas semánticos e ingeniería web.

En la Tabla IV se muestran los autores más citados en el campo de la Inteligencia de Negocio y la Inteligencia Competitiva entre 1959 y 2017.
Es importante resaltar, que dentro de los autores más citados, Arnott, D. es el único autor que aparece dentro de los autores referentes para ambos enfoques y que Yeoh, W., Rizzi, S. y Arnott, D. son los únicos autores que se encuentran dentro de los más productivos y más citados a la vez. Por su parte, Arnott, D. (profesor de la Universidad de Monas) desarrolla sus principales investigaciones en el campo de la Inteligencia de Negocio y sistemas de decisiones.

En línea con lo anterior, la Tabla V presenta las organizaciones más productivas según Scopus. La Universidad de Minho, Academia de Ciencias China, Universidad de Stuttgart, Universidad de Monash y Universidad de Ottawa, son de las organizaciones más productivas considerando su producción tanto en el área de Inteligencia de Negocio como la de Inteligencia Competitiva. En este sentido, si consideramos la línea de Inteligencia de Negocio, la Universidad de Alicante y Universidad Tecnológica de Sídney aparecen dentro de las más productivas, mientras que en el ámbito de la Inteligencia Competitiva destacan la Instituto Tecnológico de Harbin, Universidad de Tsinghua, Universidad de Zhejiang, Universidad de la Ciudad de Hong Kong y Universidad de Toronto.

Por último, es importante resaltar que las organizaciones incluidas en la Consulta (C1) tienen presencia en el resto de consultas, aspecto que refleja la coexistencia y desarrollo de ambos enfoques de inteligencia, situación que se verifica a través del análisis de las estructuras intelectuales en las siguientes secciones.

Tabla IV. Autores más citados en el campo de la inteligencia de 1959 a 2017

\begin{tabular}{|c|c|}
\hline $\begin{array}{l}\text { (C2) BI }(\mathrm{n}=33.295) \\
\text { (Citas) Autor(es) }\end{array}$ & $\begin{array}{l}\text { (C3) CI }(n=54.137) \\
\text { (Citas) Autor(es) }\end{array}$ \\
\hline (1.226) Chiang, R.H.L. & (837) Martínez, V. \\
\hline (1.132) Storey, V.C. & $\begin{array}{l}\text { (811) Alcock, J.R.; Angus, J.P.; Baines, T.S.; Basti, M.; Braganza, A.; } \\
\text { Cousens, A.; Evans, S.; Greenough, R.; Irving, P.; Johnson, M.; Kingston, } \\
\text { J.; Lightfoot, H.W.; Lockett, H.; Michele, P.; Neely, A.; Peppard, J.; Roy, R.; } \\
\text { Shehab, E.; Tiwari, A.; Tranfield, D.; Walton, I.M.; Wilson, H. }\end{array}$ \\
\hline (841) Wixom, B.H. & (725) Van Der Aalst, W.M.P \\
\hline (665) Dayal, U. & (618) Shankar, R.. \\
\hline (643) Liu, H. & (578) Petrovic, J.; Read, C.; Satyapal, S.; Thomas, G. \\
\hline $\begin{array}{l}\text { (605) Thusoo, A.; Sarma, J.S.; Jain, N.; } \\
\text { Shao, Z.; Murthy, R. }\end{array}$ & (552) Ravichandran, T. \\
\hline (541) Arnott, D. & (550) Grover, V. \\
\hline (513) Pervan, G. & (537) Bhatt, G.D. \\
\hline (481) Watson, H.J. & (534) Arnott, D. \\
\hline (474) Rizzi, S. & (525) Chau, P.Y.K.; Kuan, K.K.Y. \\
\hline
\end{tabular}


Tabla V. Organizaciones más productivas en el campo de la inteligencia de 1959 a 2017

\begin{tabular}{|c|c|c|}
\hline $\begin{array}{l}\text { (C1) BI+CI }(n=10.316) \\
\text { (Publicaciones) Organización }\end{array}$ & $\begin{array}{l}\text { (C2) BI }(n=6.031) \\
\text { (Publicaciones) Organización }\end{array}$ & $\begin{array}{l}\text { (C3) CI }(n=5.920) \\
\text { (Publicaciones) Organización }\end{array}$ \\
\hline (72) Universidad de Minho & (67) Universidad de Minho & (33) Universidad de Monash \\
\hline (64) Laboratorios Hewlett Packard & (60) Laboratorios Hewlett Packard & (33) Instituto Tecnológico de Harbin \\
\hline (59) Academia de Ciencias China & (46) Academia de Ciencias China & (33) Universidad de Minho \\
\hline (49) Universidad de Stuttgart & (45) Universidad de St. Gallen & (29) Universidad de Tsinghua \\
\hline (48) Universidad de Monash & (44) Universidad de Stuttgart & (28) Universidad de Ottawa \\
\hline (48) Universidad de Ottawa & (41) Universidad de Monash & (26) Universidad de Zhejiang \\
\hline (47) Universidad de Tsinghua & (38) Universidad de Alicante & (26) Universidad de la Ciudad de Hong Kong \\
\hline (46) Universidad de St. Gallen & (36) Universidad Tecnológica de Sídney & (26) Universidad de Stuttgart \\
\hline $\begin{array}{l}\text { (43) Centro de Investigación IBM } \\
\text { Thomas J. Watson }\end{array}$ & (36) Universidad de Ottawa & (24) Academia de Ciencias China \\
\hline (43) Universidad de Aalborg & (35) Universidad Aalborg & (22) Universidad de Toronto \\
\hline
\end{tabular}

\subsection{Análisis de publicaciones más citadas}

En vista de los resultados previos, a continuación, se consideran las publicaciones más citadas y relevantes para cada enfoque de inteligencia a través del uso del $h$-index y $H$-Classics (Martínez y otros, 2014; Hirsch, 2005).

Para ello, se analiza el peso de las publicaciones más citadas en el desarrollo de los enfoques de Inteligencia de Negocio e Inteligencia Competitiva desde la combinación de dos indicadores: $h$-index y H-Classics (Martínez y otros, 2015; Ale Ebrahim y otros, 2014). Por un lado, el $h$-index permite evaluar tanto la productividad como el impacto de un grupo de publicaciones (principalmente de áreas de conocimiento cercanas), a través de la relación que existe el número de artículos y las citas de estos (Hirsch, 2005). Por el otro lado, el H-Classics sirve de indicador de control, ya que permite estandarizar el procedimiento de búsqueda de las citaciones de los artículos incluidos en el $h$-index
(Martínez y otros, 2014). La combinación de ambos índices disminuye la discrepancia que puede existir entre los artículos más citados y los que aún no han alcanzado el mismo nivel, estableciendo un punto de referencia común para las publicaciones referentes dentro de un área de conocimiento.

En este sentido, se utilizó como referencia el $h$-index indicado por Scopus para la consulta C1 por ser la más amplia e inclusiva para la identificación de las publicaciones más relevantes en el desarrollo de los enfoques de inteligencia. Según el $h$-index de la consulta $\mathrm{C} 1$, esta se compone por 113 publicaciones (82 artículos, 15 publicaciones en congresos, 10 revisiones y 6 libros) y su distribución, incluyendo el número de citas acumuladas, se muestra en la Figura 3.

La distribución de publicaciones muestra un patrón en función del enfoque, las correspondientes a Inteligencia de Negocio representan el 35\% y van del año 2002 al 2013, mientras que las correspondientes a Inteligencia Competitiva representan el

Figura 3. Distribución de publicaciones más relevante según h-index de Scopus en consulta C1

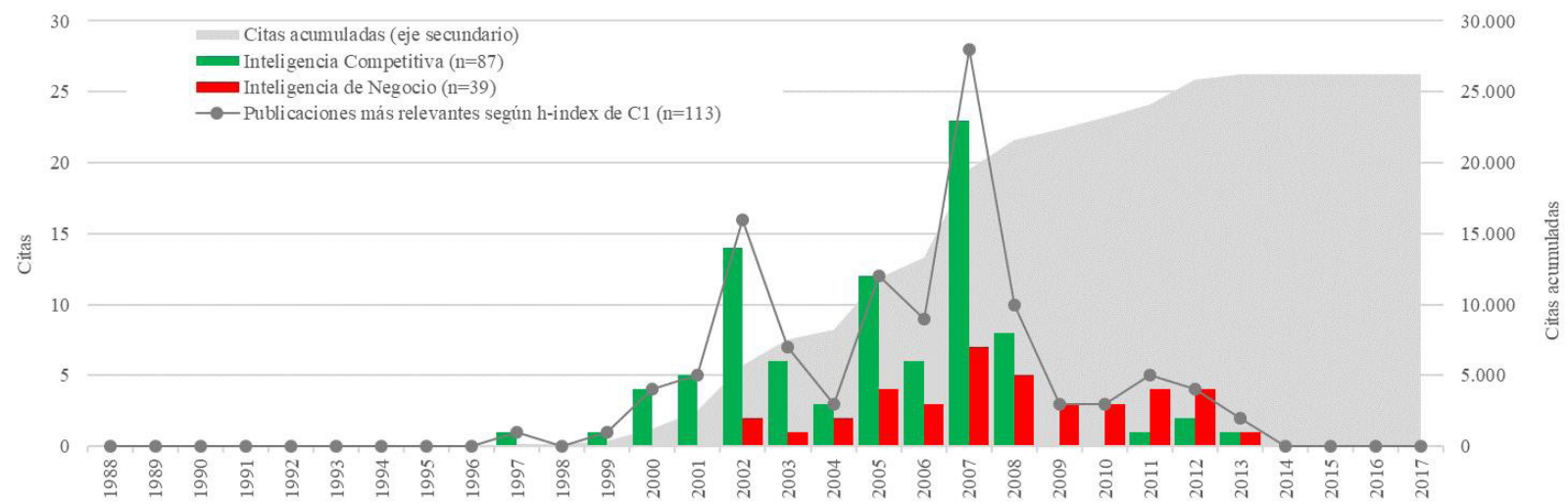


$77 \%$ y van de 1997 a 2013. En términos de citas, las publicaciones identificadas acumulan 26.229 referencias, lo que representa el 33\% de las citas totales recogidas en la consulta $\mathrm{C} 1$.

Estos aspectos, coinciden con la evolución en términos de producción y citas expresados en puntos anteriores y se mantienen una vez transcurrido un periodo ventana de siete años, lo que sugiere que la evolución y consolidación de estas publicaciones como parte del núcleo de los enfoques de Inteligencia de Negocio e Inteligencia Competitiva (Wang, 2013).

De igual forma, la productividad de los autores es más homogénea, ya que de los 160 autores que respaldan las publicaciones más relevantes, tan solo 14 de ellos cuentan con dos publicaciones, mientras que el resto registra una única publicación, siendo los autores más productivos: Arnott, D., Chen, $H_{\text {., }}$ Jain, N., Jindal, N., Liu, B., Liu, H., Murthy, R., Pervan, G., Schultze, U., Shankar, R., Shao, Z., Thusoo, A., Van Der Aalst, W.M.P. y Wixom, B.H.

En términos de distribución geográfica del autor en correspondencia, Estados Unidos mantiene la posición principal con 57\% (65 publicaciones), seguida por Australia, China y Holanda, con 6,2\%, $4,4 \%$ y $3,5 \%$ ( 7,5 y 4 publicaciones) respectivamente. Esto contrasta con los resultados iniciales donde países como Alemania, Reino Unido y Canadá aparecían en las primeras posiciones.

En este sentido, las organizaciones más productivas dentro de las publicaciones más relevantes son Universidad Tecnológica de Eindhoven (4 publicaciones), Universidad de New York (3 publicaciones), Universidad de California Berkeley (3 publicaciones), Universidad de Curtin (3 publicaciones), Universidad de Gent (3 publicaciones) y Escuela de Negocios Leonard N. Stern (3 publicaciones). Es importante resaltar, que estas organizaciones no se encuentran dentro de las organizaciones más productivas incluidas en la sección anterior, aspecto que refleja una diferencia entre la productividad y la relevancia en términos de citas.

Por otro lado, las principales áreas de conocimiento cubiertas por Inteligencia de Negocio e Inteligencia Competitiva, desde 1959 a 2017 coinciden con las identificadas en puntos anteriores, aunque su prioridad varia ligeramente posicionado Business, Management and Account delante de Computer Science, Engineering, Decision Sciences y Mathematics. Otra situación distinta ocurre con las fuentes de información, que pasan de ser principalmente repositorios de congresos para convertirse en las siguientes revistas: Strategic Management Journal, Journal of Operations Management, MIS Quarterly: Management Information Systems, Information and Management, Journal of Management Information Systems, Management Science y Decision Support Systems.
Finalmente, el listado $\mathrm{H}$-Classics se presenta en la Tabla A.I del Apéndice, donde se pueden observar los artículos más relevantes para el desarrollo de ambos enfoques de inteligencia, la afiliación del autor en correspondencia y el país de origen, entre otros datos.

\subsection{Análisis del mapa de evolución de los en- foques de inteligencia}

Para visualizar y analizar los temas de investigación más representativos de los enfoques de Inteligencia de Negocio e Inteligencia Competitiva, así como su relación, composición y evolución (Calero-Medina y Noyons, 2008), se han dispuesto dos mapas científicos utilizando como base los documentos extraídos de Scopus entre 1959 y 2017 y VOSviewer como herramienta bibliométrica para su confección.

VOSviewer permite agrupar a través del uso de algoritmos las palabras claves incluidas por los autores en sus publicaciones y en las bases de datos en lo que se ha denominado temas de investigación. Además, este software permite visualizar la relación que existe entre los temas de investigación, de tal manera que es posible identificar relaciones que a simple vista no sería posible. Asimismo, si un tema está representado junto a otro, significa que ambos comparten elementos en común, pero además estos pueden estar unidos, lo que representaría una relación directa y estrecha. El tamaño de los círculos de cada tema es proporcional al número de documentos que incluye, mientras que el grosor representa el número de documentos que comparten.

En la Figura 4 se muestra el mapa científico o de co-ocurrencias para los enfoques de inteligencia. En este sentido, las líneas de investigación identificadas se representan en forma de círculos y tienen una ocurrencia mínima de 25 veces dentro de la base de datos confeccionada para este análisis. Las líneas de investigación con el mismo color se agrupan en lo que se denomina Cluster y agrupan los conceptos que guardan estrecha relación por encontrarse contenidos en las mismas publicaciones. Por ejemplo, las líneas de investigación con color rojo están estrechamente relacionadas con el enfoque Inteligencia de Negocio, aunque también pueden guardar vínculos con otras líneas de otros grupos. Además, el grosor de la línea de conexión entre dos conceptos cualesquiera indica la fuerza de la relación que existe entre ambos, por ejemplo, la fuerza del enlace (relación) entre business intelligence y decision making process es 621 y representa una línea gruesa, lo que habla de una fuerte relación en el desarrollo de ambos conceptos. 
Antes de analizar la estructura intelectual de cada enfoque, se presentan en la Tabla VI, las principales líneas de investigación identificadas en base a los términos más desarrollados en la base de datos confeccionada para esta investigación, los mismos que son utilizados para desarrollar los mapas de co-ocurrencia más adelante.

Tomando como referencia la Tabla VI, la línea de investigación con mayor ocurrencia es competitive intelligence, seguida por business intelligence, data mining, decision making process e information analysis. Estas líneas de investigación concentran más del $40 \%$ de las ocurrencias y recogen la esencia del concepto de inteligencia, ya que van desde el tratamiento de los datos hasta el proceso de toma de decisión pasando por el análisis de información. Además, concentran más del 35\% de las relaciones, situación que manifiesta la interacción que existe entre los enfoques de inteligencia analizados y las principales líneas de investigación.

De acuerdo con esto, la estructura intelectual de los enfoques de Inteligencia de Negocio e Inteligencia Competitiva concentra 50 líneas de investigación y 1.212 vínculos directos, con una fuerza total de 66.677, repartidos en dos Cluster: Inteligencia de Negocio (25 elementos) e Inteligencia Competitiva (25 elementos).

El Cluster Inteligencia Competitiva concentra 16.702 ocurrencias, 88 vínculos con líneas pertenecientes al mismo grupo y 73 a temas externos, con una fuerza de 21.040 y 15.817 vínculos respectivamente.

Tabla VI. Principales líneas de investigación para los enfoques de inteligencia

\begin{tabular}{|c|c|c|c|}
\hline No. & Línea de investigación & Ocurrencias ( $\%$ total) & Vínculos (\% total) \\
\hline 1 & competitive intelligence & $5.475(16,17 \%)$ & $16.486(12,36 \%)$ \\
\hline 2 & business intelligence & $2.596(7,67 \%)$ & $9.967(7,47 \%)$ \\
\hline 3 & data mining & $1.971(5,82 \%)$ & $8.267(6,19 \%)$ \\
\hline 4 & decision making process & $1.411(4,17 \%)$ & $6.338(4,75 \%)$ \\
\hline 5 & information analysis & $1.230(3,63 \%)$ & $5.717(4,28 \%)$ \\
\hline 6 & industrial management & $1.215(3,59 \%)$ & $3.984(2,98 \%)$ \\
\hline 7 & information management & $1.195(3,53 \%)$ & $5.156(3,86 \%)$ \\
\hline 8 & strategic management & $1.126(3,33 \%)$ & $3.840(2,87 \%)$ \\
\hline 9 & data warehouse & $1.008(2,98 \%)$ & $4.488(3,36 \%)$ \\
\hline 10 & web services & $985(2,91 \%)$ & $4.069(3,05 \%)$ \\
\hline 11 & information systems & $952(2,81 \%)$ & $4.131(3,09 \%)$ \\
\hline 12 & data management & $930(2,75 \%)$ & $4.128(3,09 \%)$ \\
\hline 13 & knowledge management & $731(2,61 \%)$ & $2.907(2,17 \%)$ \\
\hline 14 & market research & $692(2,04 \%)$ & $2.358(1,76 \%)$ \\
\hline 15 & decision support system & $686(2,03 \%)$ & $3.469(2,60 \%)$ \\
\hline 16 & business management & $651(1,92 \%)$ & $2.820(2,11 \%)$ \\
\hline 17 & information technology & $622(1,84 \%)$ & $2.431(1,82 \%)$ \\
\hline 18 & customer relationship management & $603(1,78 \%)$ & $2.353(1,76 \%)$ \\
\hline 19 & data analytics & $583(1,72 \%)$ & $2.610(1,95 \%)$ \\
\hline 20 & artificial intelligence & $541(1,60 \%)$ & $2.644(1,98 \%)$ \\
\hline 21 & quality management & $533(1,57 \%)$ & $1.869(1,40 \%)$ \\
\hline 22 & performance management & $464(1,37 \%)$ & $1.685(1,26 \%)$ \\
\hline 23 & big data & $452(1,34 \%)$ & $1.918(1,43 \%)$ \\
\hline 24 & business intelligence system & $437(1,29 \%)$ & $1.918(1,43 \%)$ \\
\hline 25 & product design and development & $419(1,24 \%)$ & $1.415(1,06 \%)$ \\
\hline 26 & e-business & $418(1,23 \%)$ & $1.709(1,28 \%)$ \\
\hline 27 & database systems & $399(1,18 \%)$ & $1.738(1,30 \%)$ \\
\hline
\end{tabular}




\begin{tabular}{|c|c|c|c|}
\hline No. & Línea de investigación & Ocurrencias (\% total) & Vínculos (\% total) \\
\hline 28 & enterprise resource management & $384(1,13 \%)$ & $1.651(1,23 \%)$ \\
\hline 29 & knowledge based systems & $369(1,09 \%)$ & $1.629(1,22 \%)$ \\
\hline 30 & expert systems & $318(0,94 \%)$ & $1.448(1,08 \%)$ \\
\hline 31 & supply chain management & $301(0,89 \%)$ & $1.221(0,91 \%)$ \\
\hline 32 & computer programming languages & $294(0,87 \%)$ & $1.269(0,95 \%)$ \\
\hline 33 & online analytical processing & $282(0,83 \%)$ & $1.432(1,07 \%)$ \\
\hline 34 & social media and networking & $282(0,83 \%)$ & $1.120(0,83 \%)$ \\
\hline 35 & sustainable development & $278(0,82 \%)$ & $993(0,74 \%)$ \\
\hline 36 & data reduction & $259(0,77 \%)$ & $1.246(0,93 \%)$ \\
\hline 37 & risk management & $241(0,71 \%)$ & $881(0,66 \%)$ \\
\hline 38 & cloud computing & $240(0,71 \%)$ & $992(0,74 \%)$ \\
\hline 39 & data visualization & $234(0,69 \%)$ & $1.016(0,76 \%)$ \\
\hline 40 & algorithms & $224(0,66 \%)$ & $864(0,64 \%)$ \\
\hline 41 & business environment & $213(0,63 \%)$ & $789(0,59 \%)$ \\
\hline 42 & competitive advantage & $210(0,62 \%)$ & $846(0,63 \%)$ \\
\hline 43 & innovation & $197(0,58 \%)$ & $629(0,47 \%)$ \\
\hline 44 & machine learning & $191(0,56 \%)$ & $820(0,61 \%)$ \\
\hline 45 & research and development management & $178(0,53 \%)$ & $670(0,50 \%)$ \\
\hline 46 & information services & $176(0,52 \%)$ & $778(0,58 \%)$ \\
\hline 47 & technology transfer & $171(0,51 \%)$ & $637(0,47 \%)$ \\
\hline 48 & real time systems & $165(0,49 \%)$ & $769(0,57 \%)$ \\
\hline 49 & forecasting & $164(0,48 \%)$ & $636(0,47 \%)$ \\
\hline 50 & data privacy and security & $156(0,46 \%)$ & $603(0,45 \%)$ \\
\hline
\end{tabular}

Igualmente, dentro de la estructura intelectual del enfoque de Inteligencia Competitiva destacan por su co-ocurrencia las siguientes líneas: CI-01: competitive intelligence (5.475), CI-02: industrial management (1.215), CI-03: information management (1.195), CI-04: strategic management (1.126), CI-05: knowledge management (731), CI-06: market research (692), CI-07: business management (651), CI-08: customer relationship management (603), CI-09: quality management (533) y CI-10: performance management (464). Del mismo modo, los elementos con mayor interacción con temas incluidos dentro del grupo son: CI-01: competitive intelligence (24), CI-04: strategic management (9), CI-02: industrial management (8), CI-03: information management (5) y CI-06: market research (5), y con temas externos: CI-01: competitive intelligence (24), CI-03: information management (13), CI-07: business management (7), CI-02: industrial management (4), CI-04: strategic management (4), CI-05: knowledge management (4) y CI-08: customer relationship management (4).
Por su parte, el Cluster Inteligencia de Negocio concentra 17.150 ocurrencias, 164 vínculos con líneas pertenecientes al mismo grupo y 73 a temas externos, con una fuerza de 33.380 y 15.817 vínculos respectivamente.

Dentro de la estructura intelectual del enfoque de Inteligencia de Negocio destacan por su co-ocurrencia las siguientes líneas: BI-01: business intelligence (2.596), BI-02: data mining (1.971), BI-03: decision making process (1.411), BI-04: information analysis (1.230), BI-05: data warehouse (1.008), BI-06: web services (985), BI-07: information systems (952), BI-08: data management (930), BI-09: decision support system (686) y BI-10: information technology (622). Del mismo modo, los elementos con mayor interacción con temas incluidos dentro del grupo son: BI-02: data mining (21), BI-01: business intelligence (18), BI-03: decision making process (13), BI-04: information analysis (12) y BI05: data warehouse (12), y con temas externos: $B I-$ 01: business intelligence (12), BI-02: data mining (11), BI-03: decision making process (7), BI-04: information analysis (5) y BI-06: web services (5). 
En este sentido, podemos observar que la estructura intelectual del enfoque de Inteligencia de Negocio se encuentra constituida por líneas con el tratamiento y gestión de datos internos o transaccionales, el desarrollo y aplicación de herramientas y sistemas informáticos para el tratamiento y gestión de datos, así como la toma de decisiones. Mientras que, por su parte, la estructura intelectual de Inteligencia Competitiva se encuentra constituida por líneas relacionadas con la gestión de las organizaciones y su estrategia, la gestión de la información y el conocimiento, la mejora de la competitividad y el dominio del mercado a través de la relación con los clientes, la calidad y entorno.

Además, tomando en cuenta la Figura 4, es posible visualizar que las relaciones del enfoque business intelligence son más fuertes con los elementos incluidos dentro del propio grupo y con el tema de competitive intelligence, aspectos que contrastan con el comportamiento del enfoque de Inteligencia Competitiva que presenta un comportamiento más equilibrado en términos de relación con los elementos internos y externos. No obstante, este último enfoque presenta una menor interacción con los elementos integrados por la Inteligencia de Negocio.

Tomando como referencia lo anterior, se aprecia que existe una relación directa, complementaria y significativa entre el enfoque de Inteligencia de Negocio e Inteligencia Competitiva, lo que permite proponer la confección de un único enfoque de inteligencia que les integre bajo un mismo marco de trabajo.

Siguiendo con el análisis, en la Figura 5 se muestra el mapa científico de evolución de los enfoques de Inteligencia de Negocio e Inteligencia Competitiva, donde es posible corroborar parte de los resultados obtenidos en los puntos anteriores, donde se expone al enfoque Inteligencia Competitiva como primera aproximación de las organizaciones en el desarrollo del campo de la inteligencia y cercano a temas relacionados con estrategia, competitividad y toma de decisiones. Además, expone a la Inteligencia de Negocio delante en la escala temporal y cercana a conceptos ligados a la toma de decisiones, desde la explotación de datos a través de sistemas de inteligencia. Ambos enfoques se relacionan con el análisis de la información y grandes cantidades de datos.

Por último, el mapa de evolución refleja de igual forma que el desarrollo de la disciplina de inteligencia desde la óptica de las organizaciones y el uso inteligente de los datos, la información y el conocimiento es relativamente reciente y se encuentra en crecimiento.

Finalmente, en la Figura A.1 del Anexo se exponen los temas de investigación identificados en cada Cluster, ordenados en base al número de ocurrencias y sus vínculos con otros temas, permitiendo visualizar la relación que existe entre los enfoques de inteligencia analizados.

\section{CONCLUSIONES}

En este primer estudio bibliométrico sobre la relación entre los enfoques de Inteligencia de Negocio e Inteligencia Competitiva, se han identificado las principales líneas de investigación de cada enfoque y su relación, aportando una visión holística del concepto de inteligencia. Más de 10.316 documentos desde 1959 hasta 2017 han sido analizados, procesados y visualizados utilizando técnicas y herramientas bibliométricas.

En términos de rendimiento bibliométrico (cantidad y calidad), tanto el enfoque Inteligencia de Negocio como Inteligencia Competitiva presentan una tendencia positiva de crecimiento y consolidación como áreas de conocimiento, a pesar de las variaciones registradas durante los años 2008 y 2009. Tomando en cuenta el volumen de publicaciones y citas generadas en estos ámbitos de conocimiento, se espera que la investigación, aplicación e interés por parte de académicos y profesionales siga en aumento durante los próximos años, favoreciendo inclusive a otras áreas de conocimiento como Gestión Empresarial y Estratégica, Toma de Decisiones, Gestión del Conocimiento, Gestión y Sistemas de la Información, Redes Sociales, Internet y Big Data. Asimismo, esto refleja dos líneas generales de acción, una más cercana a los sistemas informáticos (Inteligencia de Negocio) y otra a las organizaciones (Inteligencia Competitiva), pero ambas vinculadas por la toma de decisiones y la consecución de ventajas competitivas.

En cuanto a producción y citas se refiere, se observan dos contextos. Por un lado, los autores más productivos y por otro los autores más citados. Estos contextos, más que dificultar el desarrollo del campo de inteligencia, promueven su desarrollo estableciendo por un lado el análisis en base a la producción, desde donde podemos identificar los autores, grupos de investigación y organizaciones, y por otro la aplicación, desde donde podemos identificar que temas de la inteligencia están teniendo un mayor impacto en el desarrollo y consolidación del campo de conocimiento. Siendo los autores más representativos por su producción científica y citas en conjunto, Yeoh, W., Baars, $H_{\text {., }}$ Mylopoulos, J., Golfarelli, M., Kemper, H.G., Rizzi, S., Machado, J., Arnott, D. y Schrefl, M. 


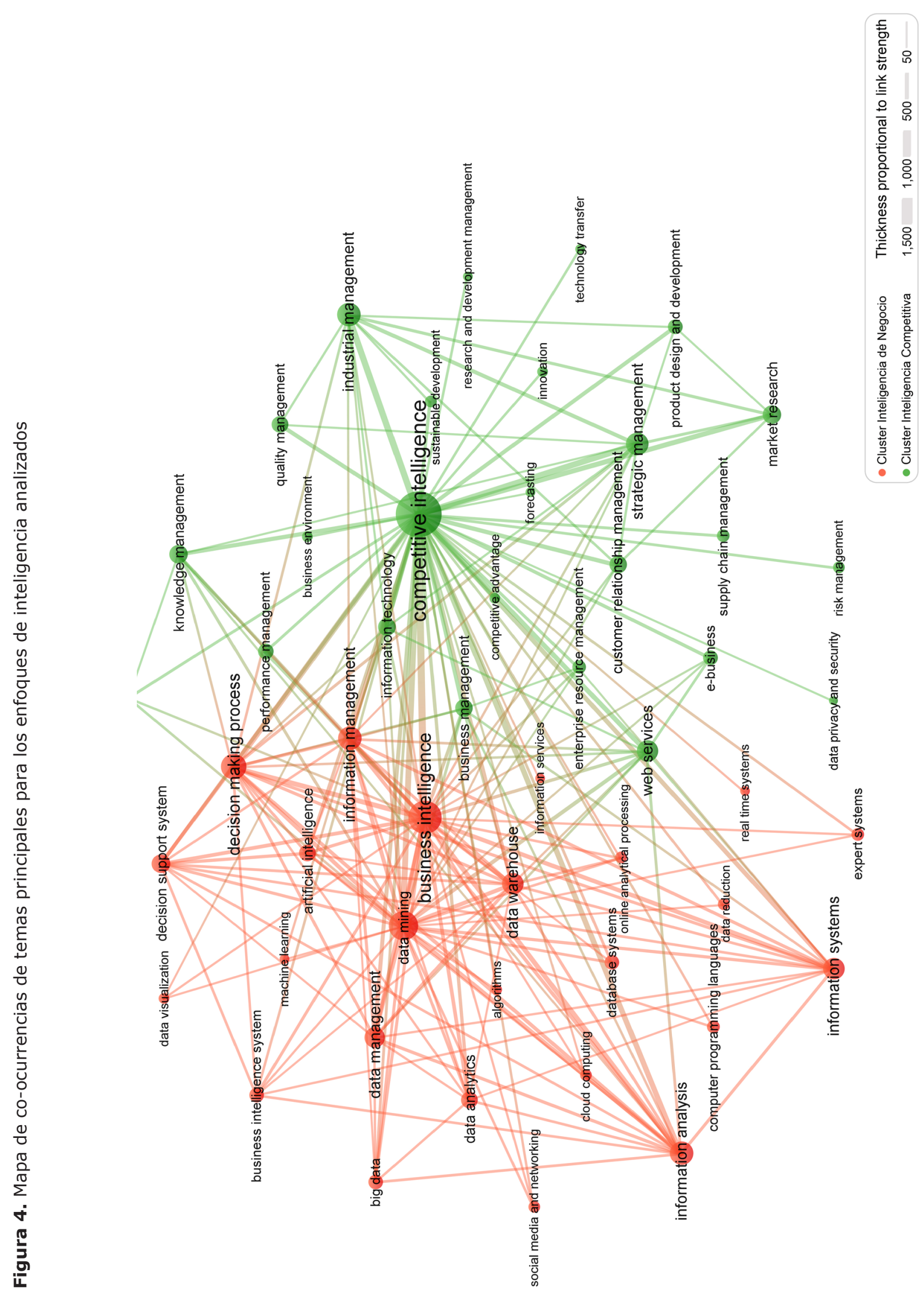




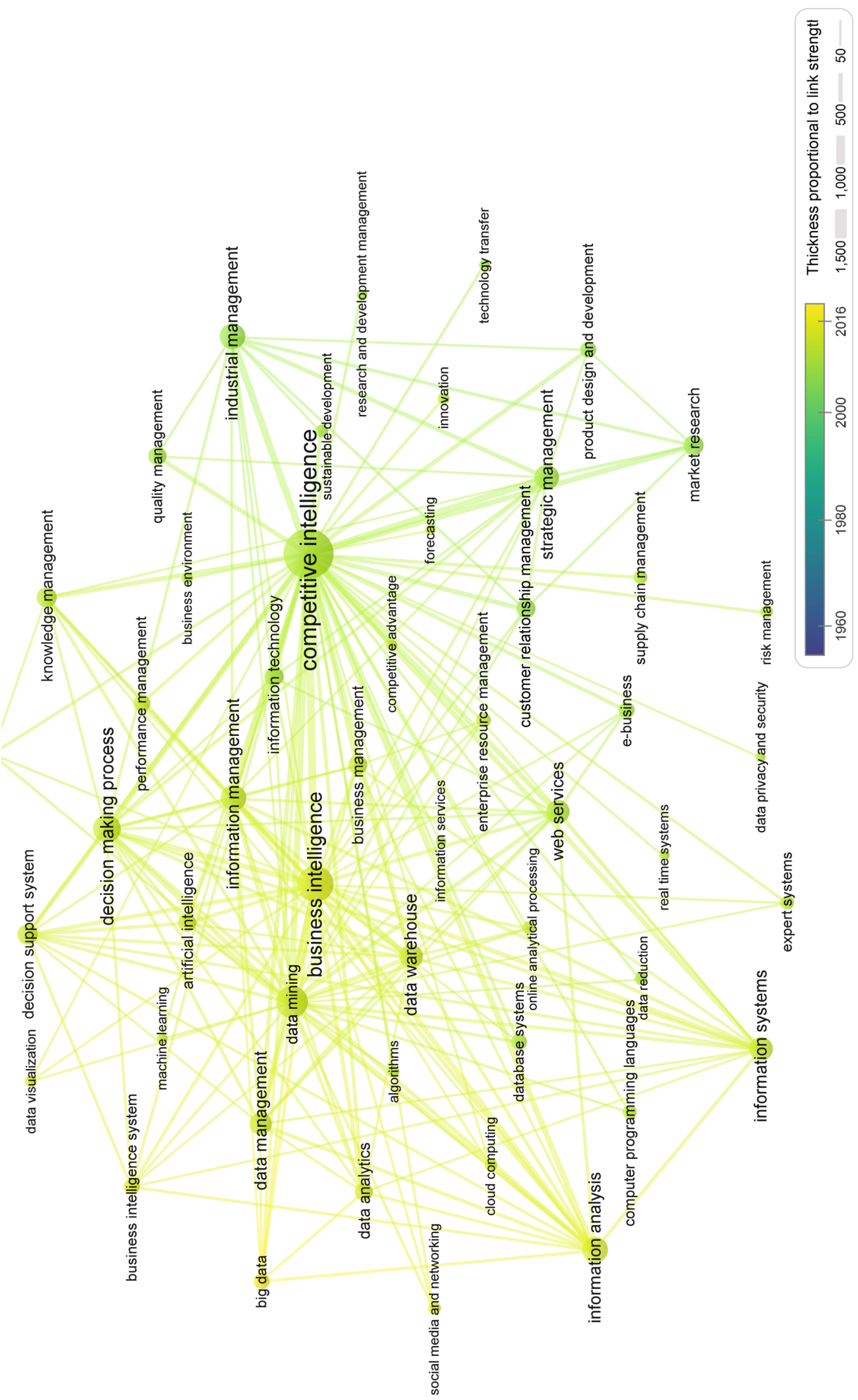


En términos de distribución geográfica y organizaciones, los resultados reflejan en cierta forma la capacidad productiva en términos académicos y empresariales, que tienen actualmente países como Estados Unidos, China, Alemania y Reino Unido, entre otros, donde la Universidad de Minho, Academia de Ciencias China, Universidad de Stuttgart, Universidad de Monash y Universidad de Ottawa, destacan por ser las organizaciones más productivas para ambos enfoques de inteligencia. Sin embargo, se puede resaltar el trabajo que presentan países como España, Portugal y Brasil en el desarrollo de estas áreas de conocimiento y que les posicionan en lugares dentro de los países más productivos. En este sentido, si consideramos la línea de Inteligencia de Negocio, la Universidad de Alicante y Universidad Tecnológica de Sídney aparecen dentro de las más productivas, mientras que en el ámbito de la Inteligencia Competitiva destacan la Instituto Tecnológico de Harbin, Universidad de Tsinghua, Universidad de Zhejiang, Universidad de la Ciudad de Hong Kong y Universidad de Toronto.

Otro aspecto significativo del análisis bibliométrico es la importancia que tienen las fuentes de información en el proceso de difusión del conocimiento. Las fuentes más activas en el campo de la inteligencia han ganado prestigio en la comunidad científica, que promueve la investigación, el desarrollo y la implementación continuos del concepto y sus principales líneas de investigación, siendo Lecture Notes in Computer Science (incluyendo las subseries Lecture Notes in Artificial Intelligence y Lecture Notes in Bioinformatics), Lecture Notes in Business Information Processing y ACM International Conference Proceedings Series, las más utilizadas. Además, es importante resaltar que estas fuentes de información cubren ámbitos de conocimiento relevantes para el desarrollo de la academia, empresa y administración, siendo Computer Science, Engineering, Business, Management and Accounting $y$ Decision Sciences, las más productivas.

Igualmente, las publicaciones que conforman actualmente el núcleo del campo de inteligencia presentan una tendencia de equilibrio entre ambos enfoques, lo que refuerza la coexistencia de éstos dentro de la organización. Además, dentro de los autores más productivos y citados se encuentran Arnott, D., Chen, H., Jain, N., Jindal, N., Liu, B., Liu, H., Murthy, R., Pervan, G., Schultze, U., Shankar, R., Shao, Z., Thusoo, A., Van Der Aalst, W.M.P. y Wixom, B.H., situación que coincide con la productividad y calidad antes descrita.

Basado en los resultados del análisis bibliométrico, fue posible obtener una visión general del desarrollo y la estructura intelectual del enfoque de Inteligencia de Negocio e Inteligencia Competitiva desde 1959 hasta 2017.
La evaluación de la estructura intelectual del concepto de inteligencia a través del análisis del mapa científico, confirma la coexistencia del enfoque de Inteligencia de Negocio e Inteligencia Competitiva, ya que se observa como comparten temas de investigación, pero a su vez cada uno cuenta con elementos propios que resultan necesarios y sinérgicos para el desarrollo del otro enfoque, y viceversa. Dentro de las líneas que vinculan ambos enfoques, se pueden resaltar cinco áreas temáticas, que se prevé promoverán el crecimiento en los próximos años atendiendo a la intensidad de éstas y su transversalidad hacia otras áreas de conocimiento:

- Toma de decisiones e inteligencia (decision making process, decision support system y artificial intelligence). Teniendo presente que el proceso de búsqueda, organización, valorización, análisis y recopilación de información en las organizaciones es complejo, la inteligencia artificial y el aprendizaje automático aportarán el dinamismo y velocidad que las organizaciones necesitan. No obstante, para ello, será necesario que los desarrollos estén alineados con las necesidades y estrategias de las organizaciones, atendiendo a su dimensión, capacidad y cultura.

- Gestión de la información y el conocimiento (information analysis, information management y knowledge management). Para asegurar la inteligencia, antes deben asegurarse los datos, la información y el conocimiento, por lo que, tanto organizaciones como profesionales buscarán asegurar y adaptar sus capacidades en torno a la gestión de los datos, la información y el conocimiento para evitar caer en problemas de sobrecarga de datos e información o en pérdidas de conocimiento. Además, con la evolución de las tecnologías de la información, esta temática se verá afectada.

- Tecnologías de la información (information system, information technology y enterprise resource management). Dada la gran cantidad de datos e información disponibles dentro y fuera de la organización, la necesidad de contar con aplicaciones y sistemas que permitan organizar, gestionar y mantenerlos resulta crítico y necesario para las organizaciones. En este sentido, la inteligencia ayuda a las organizaciones a comprender y organizar cómo deben ser esas soluciones para mejorar su competitividad.

- Gestión y explotación de datos (datawarehouse, datamining, business analytics, business intelligence systems y big data). En línea con las observaciones anteriores, es posible mencionar que las organizaciones requieren de muchos más recursos para encontrar los datos o la información que están buscando, especialmente cuando hablamos de organizaciones con gran- 
des estructuras o estructuras rígidas. En este sentido, los enfoques de inteligencia están facilitando el desarrollo de procesos organizacionales que dotan a la organización de habilidades y capacidades para dar respuesta a los retos en el uso, diseño y explotación de los datos.

- Comercio electrónico y redes sociales (business process manager, e-commerce y social media and networking). Hoy en día, el comercio electrónico y las redes sociales se han convertido en una de las principales fuentes de generación e intercambio de datos e información. La forma en que se producen, almacenan y distribuyen estos datos e información aún no es homogénea, situación que resulta una oportunidad desde el punto de vista de la inteligencia. A través de la aplicación de los enfoques de inteligencia se podrá visualizar la forma en que los usuarios buscan, adquieren y utilizan estos datos e información, incluyendo el diseño que tendrán los sistemas que los gestionarán.

Asimismo, resultado del análisis en conjunto, es posible visualizar que tanto la comunidad académica y científica como la empresarial ligan los enfoques de Inteligencia de Negocio e Inteligencia Competitiva a la competitividad de las organizaciones y al desarrollo de su estrategia, buscando sentar las bases para la construcción de organizaciones inteligentes, ágiles, flexibles y con capacidades avanzadas para la definición, recopilación, análisis, explotación y diseminación de información, independientemente del sector o mercado en el que se desarrollen. Al mismo tiempo, esta integración busca un enfoque holístico para todas las áreas y niveles de la organización, para facilitar con ello la generación de un conocimiento organizativo propio y de alto valor, que permita identificar y aprovechar oportunidades en todos los aspectos.

En términos de evolución, uno de los aspectos más relevantes es el crecimiento que está teniendo el enfoque Inteligencia de Negocio en principio resultado del empuje que ejerce el enfoque de Inteligencia Competitiva en las organizaciones por disponer de más y mejor información para la toma de decisiones y el cambio constante que se está desarrollando en el mundo de las tecnologías de la información y la captación de datos. Esta situación generará un efecto sinérgico entre ambos enfoques, manteniendo la coexistencia entre ambos y su crecimiento en el tiempo.

En este sentido, la Inteligencia de Negocio junto con sus principales líneas de investigación serán seguramente los que crezcan más rápidamente en los próximos años, siendo que están estrechamente relacionados con áreas temáticas como inteligencia artificial y aprendizaje automático, gestión de datos e información, internet y servicios avanzados. Este potencial de crecimiento está relacionado con la creciente necesidad de analizar y utilizar grandes cantidades de datos. No obstante, es importante destacar que el enfoque general de la Inteligencia de Negocio se relaciona únicamente con el desarrollo e implementación de las tecnologías de la información, y no particularmente con aspectos de la organización.

Por su parte, la Inteligencia Competitiva mantendrá su impacto en la literatura y el interés entre los investigadores y los profesionales, siendo que se encuentra estrechamente relacionada con otras áreas temáticas como la gestión de la información y el conocimiento, la toma de decisiones y la estrategia empresarial. Además, la Inteligencia Competitiva actuará de vínculo entre la organización y la Inteligencia de Negocio, adecuando el funcionamiento de la organización.

Por último, este trabajo permite tanto a investigadores como a profesionales de las áreas de la información, informática y administración, principalmente, conocer los elementos principales de ambos enfoques en la búsqueda de una inteligencia integral. Además, ayuda a definir nuevas líneas de investigación que permitan apoyar el crecimiento y consolidación de estas áreas de conocimiento, pudiendo resaltar como futuras líneas de investigación en el ámbito de la inteligencia las siguientes: la identificación de redes entre autores, el desarrollo y aplicación de la Inteligencia de Negocio e Inteligencia Competitiva en las organizaciones, la posibilidad de integrar ambos enfoques de inteligencia bajo un único marco de referencia que permita evaluar la madurez de las organizaciones y la integración de otras áreas de conocimiento como: ciberseguridad, blockchain, web 2.0, entre otros.

\section{AGRADECIMIENTOS}

Los autores J. R. López-Robles, N. K. Gamboa-Rosales y H. Gamboa-Rosales agradecen el apoyo del CONACYT-Consejo Nacional de Ciencia y Tecnología (México) y la DGRI-Dirección General de Relaciones Exteriores (México) para la realización de este estudio. Dra. Igone Porto Gómez agradece el apoyo financiero IT885-16 recibido por el Departamento de Cultura y Política Lingüística del Gobierno Vasco.

\section{ACNOWLEDGEMENTS}

The authors J. R. López-Robles, N. K. GamboaRosales y H. Gamboa-Rosales acknowledge the support by the CONACYT-Consejo Nacional de Ciencia y Tecnología (Mexico) and DGRI-Dirección General de Relaciones Exteriores (México) to carry out this study. PhD Igone Porto Gómez acknowledges financial support from the Basque Government's Department of Education, Language Policy and Culture (Grant Number IT885-16). 


\section{REFERENCIAS}

Abrahams, A. S.; Jiao, J.; Wang, G. A.; Fan, W. G. (2012). Vehicle defect discovery from social media. Decision Support Systems, 54 (1), 87-97. https://doi. org/10.1016/j.dss.2012.04.005

Ale Ebrahim, N.; Salehi, H.; Embi, M. A.; Habibi, F.; Gholizadeh, H.; Motahar, S. M. (2014). Visibility and citation impact. International Education Studies, 7 (4), 120125. https://doi.org/10.5539/ies.v7n4p120

Alonso, S.; Cabrerizo, F. J.; Herrera-Viedma, E.; Herrera, F. (2009). h-Index: A review focused in its variants, computation and standardization for different scientific fields. Journal of Informetrics, 3 (4), 273-289. https:// doi.org/10.1016/j.joi.2009.04.001

Arguimbau-Vivó, L.; Fuentes-Pujol, E.; Gallifa-Calatayud, M. (2013). Una década de investigación documental sobre cienciometría en España: análisis de los artículos de la base de datos ISOC (2000-2009). Revista Española de Documentación Científica, 36 (2), en007. http://dx.doi.org/10.3989/redc.2013.2.907

Bullinger, H. J. (2005). Realizing Enterprise Intelligence A Deeper Discussion on Concepts and Consequences to Realize the Intelligent Enterprise. En: Intelligent Enterprise: Theoretical Concepts and Practical Implications, pp. 87-110. Heidelberg: Physica-Verlag Gmbh \& Co.

Cabeza-Ramírez, L. J.; Cañizares, S. M. S.; FuentesGarcía, F. J. (2018). Characterisation of the classics of entrepreneurship (1968-2016). An analysis based on Web of Science. Revista Española de Documentacion Cientifica, 41 (2), e202. https://doi.org/10.3989/ redc. 2018.2.1488

Calero-Medina, C.; Noyons, E. C. (2008). Combining mapping and citation network analysis for a better understanding of the scientific development: The case of the absorptive capacity field. Journal of Informetrics, 2 (4), 272-279. https://doi.org/10.1016/j.joi.2008.09.005

Callon, M.; Courtial, J. P.; Laville, F. (1991). Co-Word Analysis as a Tool for Describing the Network of Interactions between Basic and Technological Research - The Case of Polymer Chemistry. Scientometrics, 22 (1), 155-205. https://doi.org/10.1007/bf02019280

Cantos-Mateos, G.; Zulueta-García, M. d. I. Á.; VargasQuesada, B.; Chinchilla-Rodríguez, Z. (2013). Estudio comparativo sobre la visualización de redes de cowords a través de los descriptores del Science Citation Index y de Medline. I Congresso ISKO Espanha e Portugal/XI Congresso ISKO Espanha. http://hdl.handle. net/10261/89290

Capo, L. R. (1983). International Drug Procurement and Market Intelligence - Cuba. World Development, 11 (3), 217-222. https://doi.org/10.1016/0305$750 \times(83) 90028-1$

Carneiro, A. (2000). How does knowledge management influence innovation and competitiveness? Journal of knowledge management, 4 (2), 87-98. https://doi. org/10.1108/13673270010372242
Cleland, D. I.; King, W. R. (1975). Competitive business intelligence systems. Business Horizons, 18 (6), 1928. https://doi.org/10.1016/0007-6813(75)90036-1

Cobo, M. J.; Lopez-Herrera, A. G.; Herrera-Viedma, E.; Herrera, F. (2011). Science Mapping Software Tools: Review, Analysis, and Cooperative Study Among Tools. Journal of the American Society for Information Science and Technology, 62 (7), 1382-1402. https://doi. org/10.1002/asi.21525

Cohen, W.; Czepiec, H. (1988). The role of ethics in gathering corporate intelligence. Journal of Business Ethics, 7 (3), 199-203. https://doi.org/10.1007/BF00381868

Cuartas, G. V.; Flórez, H. G.; Ciro, A. Ú.; Trujillo, M. V. (2014). Diversidad y reconocimiento de la producción académica en los sistemas de evaluación de la investigación en Colombia. Revista Española de Documentación Científica, 37 (3), e056. https://doi.org/10.3989/ redc. 2014.3.1133

Desai, B. H.; Bawden, D. (1993). Competitor Intelligence in the Pharmaceutical-Industry, the Role of the Information Professional. Journal of Information Science, 19 (5), 327-338. https://doi. org/10.1177/016555159301900501

Eidizadeh, R.; Salehzadeh, R.; Esfahani, A. C. (2017). Analysing the role of business intelligence, knowledge sharing and organisational innovation on gaining competitive advantage. Journal of Workplace Learning, 29 (4), 250-267. https://doi.org/10.1108/jwl-07-2016-0070

Fleisher, C. S.; Blenkhorn, D. L. (ed.) (2003). Controversies in Competitive Intelligence: The Enduring Issues. Westport, Connecticut: Praeger.

Fuld, L. M. (1985). Competitor intelligence: how to get it, how to use it, John Wiley \& Sons Inc.

Gilad, B. (1989). The Role of Organized Competitive Intelligence in Corporate-Strategy. Columbia Journal of World Business, 24 (4), 29-35.

Gilad, B.; Gilad, T. (1985). A Systems-Approach to Business Intelligence. Business Horizons, 28 (5), 65-70. https://doi.org/10.1016/0007-6813(85)90070-9

Glanzel, W. (2003). Bibliometrics as a research field a course on theory and application of bibliometric indicators. Magyar Tudományos Akadémia, Kutatásszervezési Intézet.

Glenisson, P.; Glänzel, W.; Janssens, F.; De-Moor, B. (2005). Combining full text and bibliometric information in mapping scientific disciplines. Information Processing \& Management, 41 (6), 1548-1572. https:// doi.org/10.1016/j.ipm.2005.03.021

Glynn, M. A. (1996). Innovative genius: A framework for relating individual and organizational intelligences to innovation. Academy of Management Review, 21 (4), 10811111. https://doi.org/10.5465/AMR.1996.9704071864

González-Valiente, C. L. (2016). Investigación sobre Bibliotecología, Ciencia de la Información, e Inteligencia Em- 
presarial, a través de las presentaciones a los congresos INFO e IntEmpres: un análisis bibliométrico (20022012). Revista Española de Documentación Científica, 39 (4), e154. https://doi.org/10.3989/redc.2016.4.1377

He, Q. (1999). Knowledge discovery through co-word analysis. Library Trends, 48 (1), 133-159.

Hirsch, J. E. (2005). An index to quantify an individual's scientific research output. Proceedings of the National Academy of Sciences of the United States of America, 102 (46), 16569-16572. https://doi.org/10.1073/ pnas.0507655102

Kochen, M. (1989). Management Intelligence Systems. Advances in Computers, 28 227-278. https://doi. org/10.1016/S0065-2458(08)60049-2

Kohli, A. K.; Jaworski, B. J.; Kumar, A. (1993). Markor - A Measure of Market Orientation. Journal of Marketing Research, 30 (4), 467-477. https://doi. org/10.2307/3172691

Kostoff, R. N.; Scaller, R. R. (2001). Science and technology roadmaps. IEEE Transactions on Engineering Management, 48 (2), 132-143. https://doi. org/10.1109/17.922473

Lagar Barbosa, M. P.; Escalona Fernández, M. I. ; Pulgarín Guerrero, A. (2014). Análisis de la interdisciplinariedad en la ingeniería química universitaria española. Revista Española de Documentación Científica, 37 (1), e029. http://dx.doi.org/10.3989/redc.2014.1.1048

Leydesdorff, L.; Rafols, I. (2009). A global map of science based on the ISI subject categories. Journal of the Association for Information Science and Technology, 60 (2), 348-362. https://doi.org/10.1002/asi.20967

Lichtenthaler, E. (2003). Third generation management of technology intelligence processes. $R \& D$ Management, 33 (4), 361-375. https://doi.org/10.1111/14679310.00304

Liebowitz, J. (2006). Strategic intelligence: business intelligence, competitive intelligence, and knowledge management. Auerbach Publications.

Liu, Z.; Yin, Y.; Liu, W.; Dunford, M. (2015). Visualizing the intellectual structure and evolution of innovation systems research: a bibliometric analysis. Scientometrics, 103 (1), 135-158. https://doi.org/10.1007/ s11192-014-1517-y

López-Robles, J. R.; Otegi-Olaso, J. R.; Gamboa-Rosales, N. K.; Gamboa-Rosales, H.; Cobo, M. J. (2018a). 60 Years of Business Intelligence: A Bibliometric Review from 1958 to 2017. New Trends in Intelligent Software Methodologies, Tools and Techniques: Proceedings of the 17th International Conference SoMeT 18, pp. 395-408. IOS Press. https://doi.org/10.3233/978-161499-900-3-395

López-Robles, J. R.; Otegi-Olaso, J. R. ; Porto-Gómez, I.; Gamboa-Rosales, N. K.; Gamboa-Rosales, H.; RoblesBerumen, H. (2018b). Bibliometric Network Analysis to Identify the Intellectual Structure and Evolution of the Big Data Research Field. International Confe- rence on Intelligent Data Engineering and Automated Learning, pp. 113-120. Cham: Springer. https://doi. org/10.1007/978-3-030-03496-2_13

López-Robles, J. R.; Otegi-Olaso, J. R.; Arcos, R.; Gamboa-Rosales, N. K.; Gamboa-Rosales, H. (2018c). Mapping the structure and evolution of JISIB: A bibliometric analysis of articles published in the Journal of Intelligence Studies in Business between 2011 and 2017. Journal of Intelligence Studies in Business, 8(3), 9-21. https://ojs.hh.se/index.php/JISIB/article/ view/362

López-Robles, J. R.; Otegi-Olaso, J. R.; Porto-Gómez, I.; Cobo, M. J. (2019). 30 years of intelligence models in management and business: A bibliometric review. International Journal of Information Management, 48, 2238. https://doi.org/10.1016/j.ijinfomgt.2019.01.013

Luhn, H. P. (1958). A Business Intelligence System. IBM Journal of Research and Development, 2 (4), 314-319. https://doi.org/10.1147/rd.24.0314

Martínez-Ávila, D.; San Segundo, R.; Zurian, F. A. (2014). Retos y oportunidades en organización del conocimiento en la intersección con las tecnologías de la información. Revista Española de Documentación Científica, 37 (3), e053. https://doi.org/10.3989/redc.2014.3.1112

Martínez, M. A.; Cobo, M. J.; Herrera, M.; HerreraViedma, E. (2015). Analyzing the Scientific Evolution of Social Work Using Science Mapping. Research on Social Work Practice, 25 (2), 257-277. https://doi. org/10.1177/1049731514522101

Martínez, M. A.; Herrera, M.; López-Gijón, J.; HerreraViedma, E. (2014). H-Classics: characterizing the concept of citation classics through $\mathrm{H}$-index. Scientometrics, 98 (3), 1971-1983. https://doi.org/10.1007/ s11192-013-1155-9

Moed, H. F.; De-Bruin, R. E.; Van-Leeuwen, T. N. (1995). New bibliometric tools for the assessment of national research performance: Database description, overview of indicators and first applications. Scientometrics, 33 (3), 381-422. https://doi.org/10.1007/BF02017338

Moreno-Fernández, O.; Moreno-Crespo, P. (2016). Análisis bibliométrico de las tesis doctorales españolas indexadas con el descriptor "Sector de la educación"(1976/2014). Revista Española de Documentación Científica, 39 (3), e146. https://doi.org/10.3989/redc.2016.3.1331

Porter, A. L.; Detampel, M. J. (1995). Technology Opportunities Analysis. Technological Forecasting and Social Change, 49 (3), 237-255. https://doi. org/10.1016/0040-1625(95)00022-3

Prescott, J. (1992). The role of competitive intelligence for US competitiveness. Competitive Intelligence Review, 2 (3), 8-10. https://doi.org/10.1002/cir.3880020305

Rodriguez-Bolivar, M. P.; Alcaide-Munoz, L.; Cobo, M. J. (2018). Analyzing the scientific evolution and impact of e-Participation research in JCR journals using science mapping. International Journal of Information Management, 40 111-119. https://doi.org/10.1016/j.ijinfomgt.2017.12.011 
Rodríguez-Pallares, M. (2016). Propuesta conceptual de un modelo de gestión de contenidos y del conocimiento en la empresa radiofónica. Revista Española de Documentación Científica, 39 (2), e127. http://dx.doi. org/10.3989/redc.2016.2.1271

Salles, M. (2006). Decision making in SMEs and information requirements for competitive intelligence. Production Planning and Control, 17 (3), 229-237. https:// doi.org/10.1080/09537280500285367

Sawyer, S.; Huang, H. (2007). Conceptualizing information, technology, and people: Comparing information science and information systems literatures. Journal of the American Society for Information Science and Technology, 58 (10), 1436-1447. https://doi. org/10.1002/asi.20621

Schreiber, M. (2007). Self-citation corrections for the Hirsch index. EPL (Europhysics Letters), 78 (3), 30002. https://doi.org/10.1209/0295-5075/78/30002

Schubert, A. (2007). Successive h-indices. Scientometrics, 70 (1), 201-205.

Schwarz, J. O. (2007). Competitive Intelligence: A Field for Futurists? Futures Research Quarterly, 23 (1), 55-65.

Shujahat, M.; Hussain, S.; Javed, S.; Malik, M. I.; Thurasamy, R.; Ali, J. (2017). Strategic management model with lens of knowledge management and competitive intelligence: A review approach. VINE Journal of Information and Knowledge Management
Systems, 47 (1), 55-93. https://doi.org/10.1108/ VJIKMS-06-2016-0035

Sternitzke, C.; Bergmann, I. (2009). Similarity measures for document mapping: A comparative study on the level of an individual scientist. Scientometrics, 78 (1), 113-130. https://doi.org/10.1007/s11192-007-1961-z

Van Eck, N. J.; Waltman, L. (2010). Software survey: VOSviewer, a computer program for bibliometric mapping. Scientometrics, 84 (2), 523-538. https://doi. org/10.1007/s11192-009-0146-3

Von Krogh, G.; Ichijo, K.; Nonaka, I. (2000). Enabling knowledge creation: How to unlock the mystery of tacit knowledge and release the power of innovation, Oxford University Press on Demand.

Walker, T. D. (1994). The literature of competitive intelligence. Library Trends, 43 (2), 271-284.

Wang, J. (2013). Citation time window choice for research impact evaluation. Scientometrics, 94 (3), 851-872. https://doi.org/10.1007/s11192-012-0775-9

Zhao, D. ; Strotmann, A. (2015). Analysis and visualization of citation networks. Synthesis lectures on information concepts, retrieval, and services, 7 (1), 1-207. https:// doi.org/10.2200/S00624ED1V01Y201501ICR039

Zinkhan, G. M.; Gelb, B. D. (1985). Competitive intelligence practices of industrial marketers. Industrial Marketing Management, 14 (4), 269-275. https://doi. org/10.1016/0019-8501(85)90019-7 


\section{APÉNDICE}

Tabla A.I. H-Classics para los enfoques de Inteligencia de Negocio e Inteligencia Competitiva

\begin{tabular}{|c|c|c|c|c|c|}
\hline No. & Titulo (referencia) & $\begin{array}{l}\text { Afiliación del autor } \\
\text { principal (País) }\end{array}$ & Fuente & $\begin{array}{c}\text { Tipo de } \\
\text { documento }\end{array}$ & Citas \\
\hline 1 & $\begin{array}{l}\text { Business intelligence and analytics: } \\
\text { From big data to big impact (Chen y } \\
\text { otros, 2012) }\end{array}$ & $\begin{array}{l}\text { Universidad de Arizona } \\
\text { (Estados Unidos) }\end{array}$ & $\begin{array}{l}\text { MIS Quarterly: } \\
\text { Management } \\
\text { Information } \\
\text { Systems } \\
\end{array}$ & Artículo & 1.345 \\
\hline 2 & $\begin{array}{l}\text { State-of-the-art in product-service } \\
\text { systems (Baines y otros, 2007) }\end{array}$ & $\begin{array}{l}\text { Universidad de } \\
\text { Cranfield (Reino Unido) }\end{array}$ & $\begin{array}{l}\text { Institution of } \\
\text { Mechanical } \\
\text { Engineers, Part } \\
\text { B: Journal of } \\
\text { Engineering } \\
\text { Manufacture } \\
\end{array}$ & Artículo & 861 \\
\hline 3 & $\begin{array}{l}\text { The U.S. Department of Energy's } \\
\text { National Hydrogen Storage Project: } \\
\text { Progress towards meeting hydrogen- } \\
\text { powered vehicle requirements } \\
\text { (Satyapal y otros, 2007) }\end{array}$ & $\begin{array}{l}\text { Departamento de } \\
\text { Energía de Estados } \\
\text { Unidos (Estados } \\
\text { Unidos) }\end{array}$ & Catalysis Today & Artículo & 606 \\
\hline 4 & $\begin{array}{l}\text { A perception-based model for EDI } \\
\text { adoption in small businesses using a } \\
\text { technology-organization-environment } \\
\text { framework (Kuan y Chau, 2001) }\end{array}$ & $\begin{array}{l}\text { Universidad de } \\
\text { Michigan (Estados } \\
\text { Unidos) }\end{array}$ & $\begin{array}{l}\text { Information and } \\
\text { Management }\end{array}$ & Artículo & 551 \\
\hline 5 & $\begin{array}{l}\text { Effect of information systems } \\
\text { resources and capabilities on firm } \\
\text { performance: A resource-based } \\
\text { perspective (Ravichandran y } \\
\text { Lertwongsatien, 2005) }\end{array}$ & $\begin{array}{l}\text { Instituto Politécnico de } \\
\text { Rensselaer (Estados } \\
\text { Unidos) }\end{array}$ & $\begin{array}{l}\text { Journal of } \\
\text { Management } \\
\text { Information } \\
\text { Systems }\end{array}$ & Revisión & 548 \\
\hline 6 & $\begin{array}{l}\text { Types of information technology } \\
\text { capabilities and their role in } \\
\text { competitive advantage: An empirical } \\
\text { study (Bhatt y Grover, 2005) }\end{array}$ & $\begin{array}{l}\text { Universidad Estatal } \\
\text { Morgan en Baltimore } \\
\text { (Estados Unidos) }\end{array}$ & \begin{tabular}{|l} 
Journal of \\
Management \\
Information \\
Systems \\
\end{tabular} & Artículo & 512 \\
\hline 7 & $\begin{array}{l}\text { Conformance checking of processes } \\
\text { based on monitoring real behavior } \\
\text { (Rozinat y van der Aalst, 2008) }\end{array}$ & $\begin{array}{l}\text { Universidad de } \\
\text { Eindhoven (Holanda) }\end{array}$ & $\begin{array}{l}\text { Information } \\
\text { Systems }\end{array}$ & Artículo & 499 \\
\hline 8 & $\begin{array}{l}\text { Hive - A petabyte scale data } \\
\text { warehouse using hadoop (Thusoo y } \\
\text { otros, 2010a) }\end{array}$ & $\begin{array}{l}\text { Escuela de Negocios } \\
\text { Goizueta (Estados } \\
\text { Unidos) } \\
\end{array}$ & $\begin{array}{l}\text { Journal of } \\
\text { Operations } \\
\text { Management }\end{array}$ & Artículo & 438 \\
\hline 9 & $\begin{array}{l}\text { The influence of an integration } \\
\text { strategy on competitive capabilities } \\
\text { and business performance: An } \\
\text { exploratory study of consumer } \\
\text { products manufacturers (Rosenzweig } \\
\text { y otros, 2003) }\end{array}$ & Facebook (China) & $\begin{array}{l}\text { International } \\
\text { Conference on } \\
\text { Data Engineering }\end{array}$ & $\begin{array}{l}\text { Publicación en } \\
\text { congreso }\end{array}$ & 438 \\
\hline 10 & $\begin{array}{l}\text { Business process modelling: Review } \\
\text { and framework (Aguilar-Saven, } \\
\text { 2004) }\end{array}$ & $\begin{array}{l}\text { Universidad de } \\
\text { Linköping (Suecia) }\end{array}$ & $\begin{array}{l}\text { International } \\
\text { Journal of } \\
\text { Production } \\
\text { Economics } \\
\end{array}$ & Artículo & 431 \\
\hline 11 & $\begin{array}{l}\text { Controlling data in the cloud: } \\
\text { Outsourcing computation without } \\
\text { outsourcing control (Chow y otros, } \\
\text { 2009) }\end{array}$ & $\begin{array}{l}\text { PARC, a Xerox } \\
\text { Company (Estados } \\
\text { Unidos) }\end{array}$ & $\begin{array}{l}\text { ACM Conference } \\
\text { on Computer and } \\
\text { Communications } \\
\text { Security }\end{array}$ & $\begin{array}{l}\text { Publicación en } \\
\text { congreso }\end{array}$ & 417 \\
\hline 12 & $\begin{array}{l}\text { Antecedents of information and } \\
\text { system quality: An empirical } \\
\text { examination within the context of } \\
\text { data warehousing (Nelson y otros, } \\
\text { 2005) }\end{array}$ & $\begin{array}{l}\text { Universidad de Virginia } \\
\text { (Estados Unidos) }\end{array}$ & $\begin{array}{l}\text { Journal of } \\
\text { Management } \\
\text { Information } \\
\text { Systems }\end{array}$ & Artículo & 384 \\
\hline 13 & $\begin{array}{l}\text { An empirical investigation of KM } \\
\text { styles and their effect on corporate } \\
\text { performance (Choi y Lee, 2003) }\end{array}$ & $\begin{array}{l}\text { Instituto Avanzado de } \\
\text { Ciencia y Tecnología de } \\
\text { Corea (Corea del Sur) }\end{array}$ & $\begin{array}{l}\text { Information and } \\
\text { Management }\end{array}$ & Artículo & 365 \\
\hline 14 & $\begin{array}{l}\text { Review: IT-dependent strategic } \\
\text { initiatives and sustained competitive } \\
\text { advantage: A review and synthesis } \\
\text { of the literature (Piccoli y Ives, 2005) }\end{array}$ & $\begin{array}{l}\text { Universidad de Cornell } \\
\text { (Estados Unidos) }\end{array}$ & $\begin{array}{l}\text { MIS Quarterly: } \\
\text { Management } \\
\text { Information } \\
\text { Systems }\end{array}$ & Artículo & 364 \\
\hline
\end{tabular}




\begin{tabular}{|c|c|c|c|c|c|}
\hline No. & Titulo (referencia) & $\begin{array}{l}\text { Afiliación del autor } \\
\text { principal (País) }\end{array}$ & Fuente & $\begin{array}{c}\text { Tipo de } \\
\text { documento }\end{array}$ & Citas \\
\hline 15 & $\begin{array}{l}\text { Analysis of interactions among the } \\
\text { barriers of reverse logistics (Raci y } \\
\text { Shankar, 2005) }\end{array}$ & $\begin{array}{l}\text { Instituto Indio de } \\
\text { Tecnología de Delhi } \\
\text { (India) }\end{array}$ & $\begin{array}{l}\text { Technological } \\
\text { Forecasting and } \\
\text { Social Change } \\
\end{array}$ & Artículo & 352 \\
\hline 16 & $\begin{array}{l}\text { Measuring factors that influence } \\
\text { the success of Internet commerce } \\
\text { (Torkzadeh y Dhillon, 2002) }\end{array}$ & $\begin{array}{l}\text { Universidad de Nevada } \\
\text { (Estados Unidos) }\end{array}$ & $\begin{array}{l}\text { Information } \\
\text { Systems Research }\end{array}$ & Artículo & 336 \\
\hline 17 & \begin{tabular}{|l|l|} 
CEO characteristics and firm R\&D \\
spending (Barker y Mueller, 2002)
\end{tabular} & \begin{tabular}{|l|} 
Universidad de Kansas \\
(Estados Unidos)
\end{tabular} & $\begin{array}{l}\text { Management } \\
\text { Science } \\
\end{array}$ & Artículo & 332 \\
\hline 18 & $\begin{array}{l}\text { Knowledge relatedness and the } \\
\text { performance of multibusiness firms } \\
\text { (Tanriverdi y Venkatraman, 2005) }\end{array}$ & $\begin{array}{l}\text { Universidad de Texas } \\
\text { (Estados Unidos) }\end{array}$ & $\begin{array}{l}\text { Strategic } \\
\text { Management } \\
\text { Journal } \\
\end{array}$ & Revisión & 330 \\
\hline 19 & $\begin{array}{l}\text { Does business planning facilitate } \\
\text { the development of new ventures? } \\
\text { (Delmar y Shane, 2003) }\end{array}$ & $\begin{array}{l}\text { Escuela de Negocios de } \\
\text { Stockholm (Suecia) }\end{array}$ & $\begin{array}{l}\text { Strategic } \\
\text { Management } \\
\text { Journal }\end{array}$ & Artículo & 313 \\
\hline 20 & $\begin{array}{l}\text { Performance measures and metrics } \\
\text { in logistics and supply chain } \\
\text { management: A review of recent } \\
\text { literature (1995-2004) for research } \\
\text { and applications (Gunasekaran y } \\
\text { Kobu, 2007) } \\
\end{array}$ & $\begin{array}{l}\text { Universidad de } \\
\text { Massachusetts } \\
\text { Dartmouth (Estados } \\
\text { Unidos) }\end{array}$ & $\begin{array}{l}\text { International } \\
\text { Journal of } \\
\text { Production } \\
\text { Research }\end{array}$ & Artículo & 305 \\
\hline 21 & $\begin{array}{l}\text { Toward a model of strategic } \\
\text { outsourcing (Holcomb y Hitt, 2007) }\end{array}$ & $\begin{array}{l}\text { Universidad de Texas } \\
\text { A\&M (Estados Unidos) }\end{array}$ & $\begin{array}{l}\text { Journal of } \\
\text { Operations } \\
\text { Management }\end{array}$ & Artículo & 300 \\
\hline 22 & $\begin{array}{l}\text { When are technologies disruptive? } \\
\text { A demand-based view of the } \\
\text { emergence of competition (Adner, } \\
\text { 2002) }\end{array}$ & INSEAD (Francia) & $\begin{array}{l}\text { Strategic } \\
\text { Management } \\
\text { Journal }\end{array}$ & Artículo & 298 \\
\hline 23 & $\begin{array}{l}\text { Analyzing alternatives in reverse } \\
\text { logistics for end-of-life computers: } \\
\text { ANP and balanced scorecard } \\
\text { approach (Ravi y otros, 2005) }\end{array}$ & $\begin{array}{l}\text { Instituto Indio de } \\
\text { Tecnología de Delhi } \\
\text { (India) }\end{array}$ & $\begin{array}{l}\text { Computers } \\
\text { and Industrial } \\
\text { Engineering }\end{array}$ & Artículo & 288 \\
\hline 24 & $\begin{array}{l}\text { An overview of business intelligence } \\
\text { technology (Chaudhuri y otros, } \\
2011 \text { ) }\end{array}$ & $\begin{array}{l}\text { Microsoft (Estados } \\
\text { Unidos) }\end{array}$ & $\begin{array}{l}\text { Communications of } \\
\text { the ACM }\end{array}$ & Revisión & 283 \\
\hline 25 & $\begin{array}{l}\text { A confessional account of an } \\
\text { ethnography about knowledge work } \\
\text { (Schultze, 2000) }\end{array}$ & $\begin{array}{l}\text { Escuela de Negocios } \\
\text { Edwin L. Cox (Estados } \\
\text { Unidos) }\end{array}$ & $\begin{array}{l}\text { MIS Quarterly: } \\
\text { Management } \\
\text { Information } \\
\text { Systems }\end{array}$ & Artículo & 281 \\
\hline 26 & $\begin{array}{l}\text { Supplier development: Improving } \\
\text { supplier performance through } \\
\text { knowledge transfer (Modi y Mabert, } \\
\text { 2007) }\end{array}$ & $\begin{array}{l}\text { Universidad de Indiana } \\
\text { (Estados Unidos) }\end{array}$ & $\begin{array}{l}\text { Journal of } \\
\text { Operations } \\
\text { Management }\end{array}$ & Artículo & 277 \\
\hline 27 & $\begin{array}{l}\text { The current state of business } \\
\text { intelligence (Watson y Wixom, 2007) }\end{array}$ & $\begin{array}{l}\text { Universidad de Georgia } \\
\text { (Estados Unidos) }\end{array}$ & Computer & Artículo & 275 \\
\hline 28 & $\begin{array}{l}\text { Incumbent entry into new market } \\
\text { niches: The role of experience and } \\
\text { managerial choice in the creation of } \\
\text { dynamic capabilities (King y Tucci, } \\
\text { 2002) }\end{array}$ & $\begin{array}{l}\text { Escuela de Negocios } \\
\text { Leonard N. Stern de la } \\
\text { Universidad de Nueva } \\
\text { York (Estados Unidos) }\end{array}$ & $\begin{array}{l}\text { Management } \\
\text { Science }\end{array}$ & Artículo & 264 \\
\hline 29 & $\begin{array}{l}\text { Column-stores vs. row-stores: How } \\
\text { different are they really? (Abadi y } \\
\text { otros, 2008) }\end{array}$ & $\begin{array}{l}\text { Universidad Yale } \\
\text { (Estados Unidos) }\end{array}$ & $\begin{array}{l}\text { ACM SIGMOD } \\
\text { International } \\
\text { Conference on } \\
\text { Management of } \\
\text { Data } \\
\end{array}$ & $\begin{array}{l}\text { Publicación en } \\
\text { congreso }\end{array}$ & 263 \\
\hline 30 & $\begin{array}{l}\text { From reversed logistics to green } \\
\text { supply chains (Van Hoek, 1999) }\end{array}$ & $\begin{array}{l}\text { Universidad de Ghent } \\
\text { (Bélgica) }\end{array}$ & $\begin{array}{l}\text { Supply Chain } \\
\text { Management }\end{array}$ & Artículo & 257 \\
\hline 31 & $\begin{array}{l}\text { A critical analysis of decision support } \\
\text { systems research (Arnott y Pervan, } \\
2005 \text { ) }\end{array}$ & $\begin{array}{l}\text { Universidad de Curtin } \\
\text { (Australia) }\end{array}$ & $\begin{array}{l}\text { Journal of } \\
\text { Information } \\
\text { Technology } \\
\end{array}$ & Artículo & 251 \\
\hline 32 & $\begin{array}{l}\text { Price dispersion and differentiation } \\
\text { in online travel: An empirical } \\
\text { investigation (Clemons y otros, 2002) }\end{array}$ & $\begin{array}{l}\text { Universidad de } \\
\text { Pennsylvania (Estados } \\
\text { Unidos) }\end{array}$ & $\begin{array}{l}\text { Management } \\
\text { Science }\end{array}$ & Artículo & 250 \\
\hline 33 & $\begin{array}{l}\text { MAD skills: New analysis practices } \\
\text { for big data (Cohen y otros, 2009) }\end{array}$ & $\begin{array}{l}\text { Greenplum (Estados } \\
\text { Unidos) }\end{array}$ & VLDB Endowment & Artículo & 249 \\
\hline
\end{tabular}




\begin{tabular}{|c|c|c|c|c|c|}
\hline No. & Titulo (referencia) & $\begin{array}{l}\text { Afiliación del autor } \\
\text { principal (País) }\end{array}$ & Fuente & $\begin{array}{c}\text { Tipo de } \\
\text { documento }\end{array}$ & Citas \\
\hline 34 & $\begin{array}{l}\text { An empirical study of best practices } \\
\text { in virtual teams (Lurey y Raisinghani, } \\
\text { 2001) }\end{array}$ & $\begin{array}{l}\text { PricewaterhouseCoopers } \\
\text { LLP (Estados Unidos) }\end{array}$ & $\begin{array}{l}\text { Information and } \\
\text { Management }\end{array}$ & Artículo & 248 \\
\hline 35 & $\begin{array}{l}\text { The application of data mining } \\
\text { techniques in financial fraud detection: } \\
\text { A classification framework and an } \\
\text { academic review of literature (Ngai y } \\
\text { otros, 2011) }\end{array}$ & $\begin{array}{l}\text { Universidad Politécnica } \\
\text { de Hong Kong (China) }\end{array}$ & $\begin{array}{l}\text { Decision Support } \\
\text { Systems }\end{array}$ & Revisión & 244 \\
\hline 36 & $\begin{array}{l}\text { Reflections on "Profiting from } \\
\text { Innovation" (Teece, 2006) }\end{array}$ & $\begin{array}{l}\text { Oracle Corporation } \\
\text { (Estados Unidos) }\end{array}$ & $\begin{array}{l}\text { Supply Chain } \\
\text { Management }\end{array}$ & Artículo & 241 \\
\hline 37 & $\begin{array}{l}\text { Collaboration: The key to value } \\
\text { creation in supply chain management } \\
\text { (Horvath, 2001) }\end{array}$ & $\begin{array}{l}\text { Universidad de } \\
\text { California Berkeley } \\
\text { (Estados Unidos) }\end{array}$ & Research Policy & Artículo & 241 \\
\hline 38 & $\begin{array}{l}\text { Modeling control objectives for } \\
\text { business process compliance (Sadiq } \\
\text { y otros, 2007) }\end{array}$ & $\begin{array}{l}\text { Universidad de } \\
\text { Queensland (Australia) }\end{array}$ & $\begin{array}{l}\text { Lecture Notes } \\
\text { in Computer } \\
\text { Science (including } \\
\text { subseries Lecture } \\
\text { Notes in Artificial } \\
\text { Intelligence and } \\
\text { Lecture Notes in } \\
\text { Bioinformatics) }\end{array}$ & $\begin{array}{l}\text { Publicación en } \\
\text { congreso }\end{array}$ & 239 \\
\hline 39 & $\begin{array}{l}\text { The Influence of Business Managers' } \\
\text { IT Competence on Championing IT } \\
\text { (Bassellier y otros, 2003) }\end{array}$ & $\begin{array}{l}\text { Universidad McGill } \\
\text { (Canadá) }\end{array}$ & $\begin{array}{l}\text { Information } \\
\text { Systems Research }\end{array}$ & Artículo & 237 \\
\hline 40 & $\begin{array}{l}\text { A multi-theoretic perspective on trust } \\
\text { and power in strategic supply chains } \\
\text { (Ireland y Webb, 2007) }\end{array}$ & $\begin{array}{l}\text { Universidad de Texas } \\
\text { A\&M (Estados Unidos) }\end{array}$ & $\begin{array}{l}\text { Journal of } \\
\text { Operations } \\
\text { Management }\end{array}$ & Artículo & 235 \\
\hline 41 & $\begin{array}{l}\text { Identifying comparative sentences in } \\
\text { text documents (Jindal y Liu, 2006) }\end{array}$ & $\begin{array}{l}\text { Escuela de Negocios } \\
\text { Kellogg (Estados } \\
\text { Unidos) }\end{array}$ & $\begin{array}{l}\text { Strategic } \\
\text { Management } \\
\text { Journal }\end{array}$ & Artículo & 228 \\
\hline 42 & $\begin{array}{l}\text { Knowledge spillover in corporate } \\
\text { financing networks: Embeddedness } \\
\text { and the firm's debt performance (Uzzi } \\
\text { y Gillespie, 2002) }\end{array}$ & $\begin{array}{l}\text { Universidad de Illinois } \\
\text { (Estados Unidos) }\end{array}$ & $\begin{array}{l}\text { 29th Annual } \\
\text { International ACM } \\
\text { SIGIR Conference } \\
\text { on Research and } \\
\text { Development } \\
\text { in Information } \\
\text { Retrieval } \\
\end{array}$ & $\begin{array}{l}\text { Publicación en } \\
\text { congreso }\end{array}$ & 228 \\
\hline 43 & $\begin{array}{l}\text { Strategies for managing a portfolio } \\
\text { of alliances (Hoffmann, 2007) }\end{array}$ & $\begin{array}{l}\text { Centro Tufts para el } \\
\text { Estudio del Desarrollo } \\
\text { de Medicamentos } \\
\text { (Estados Unidos) }\end{array}$ & $\begin{array}{l}\text { Strategic } \\
\text { Management } \\
\text { Journal }\end{array}$ & Artículo & 226 \\
\hline 44 & $\begin{array}{l}\text { Economics of new oncology drug } \\
\text { development (DiMasi y Grabowski, } \\
\text { 2007) }\end{array}$ & $\begin{array}{l}\text { Universidad de } \\
\text { Economía de Viena } \\
\text { (Austria) }\end{array}$ & $\begin{array}{l}\text { Journal of Clinical } \\
\text { Oncology }\end{array}$ & Revisión & 226 \\
\hline 45 & $\begin{array}{l}\text { Social media competitive analysis } \\
\text { and text mining: A case study in the } \\
\text { pizza industry (He y otros, 2013) }\end{array}$ & $\begin{array}{l}\text { Universidad de Old } \\
\text { Dominion (Estados } \\
\text { Unidos) }\end{array}$ & $\begin{array}{l}\text { International } \\
\text { Journal of } \\
\text { Information } \\
\text { Management }\end{array}$ & Artículo & 225 \\
\hline 46 & $\begin{array}{l}\text { Trust and Reputation for Service- } \\
\text { Oriented Environments: Technologies } \\
\text { for Building Business Intelligence } \\
\text { and Consumer Confidence (Chang y } \\
\text { otros, 2006) }\end{array}$ & $\begin{array}{l}\text { Universidad de Curtin } \\
\text { (Australia) }\end{array}$ & $\begin{array}{l}\text { Trust and } \\
\text { Reputation for } \\
\text { Service-Oriented } \\
\text { Environments: } \\
\text { Technologies for } \\
\text { Building Business } \\
\text { Intelligence } \\
\text { and Consumer } \\
\text { Confidence }\end{array}$ & Libro & 209 \\
\hline
\end{tabular}




\begin{tabular}{|c|c|c|c|c|c|}
\hline No. & Titulo (referencia) & $\begin{array}{l}\text { Afiliación del autor } \\
\text { principal (País) }\end{array}$ & Fuente & $\begin{array}{c}\text { Tipo de } \\
\text { documento }\end{array}$ & Citas \\
\hline 47 & $\begin{array}{l}\text { Data warehousing and analytics } \\
\text { infrastructure at facebook (Thusoo y } \\
\text { otros, 2010b) }\end{array}$ & $\begin{array}{l}\text { Escuela de Negocios } \\
\text { Leonard N. Stern de la } \\
\text { Universidad de Nueva } \\
\text { York (Estados Unidos) }\end{array}$ & $\begin{array}{l}\text { MIS Quarterly: } \\
\text { Management } \\
\text { Information } \\
\text { Systems }\end{array}$ & Artículo & 205 \\
\hline 48 & $\begin{array}{l}\text { Eight key issues for the decision } \\
\text { support systems discipline (Arnott y } \\
\text { Pervan, 2008) }\end{array}$ & $\begin{array}{l}\text { Facebook (Estados } \\
\text { Unidos) }\end{array}$ & $\begin{array}{l}\text { ACM SIGMOD } \\
\text { International } \\
\text { Conference on } \\
\text { Management of } \\
\text { Data }\end{array}$ & $\begin{array}{l}\text { Publicación en } \\
\text { congreso }\end{array}$ & 205 \\
\hline 49 & $\begin{array}{l}\text { Vicious and virtuous circles in the } \\
\text { management of knowledge: The case } \\
\text { of Infosys Technologies (Garud y } \\
\text { Kumaraswamy, 2005) }\end{array}$ & $\begin{array}{l}\text { Universidad de Monash } \\
\text { (Australia) }\end{array}$ & $\begin{array}{l}\text { Decision Support } \\
\text { Systems }\end{array}$ & Artículo & 205 \\
\hline 50 & $\begin{array}{l}\text { Measuring the effects of business } \\
\text { intelligence systems: The } \\
\text { relationship between business } \\
\text { process and organizational } \\
\text { performance (Elbashir y otros, 2008) }\end{array}$ & $\begin{array}{l}\text { Universidad de } \\
\text { Queensland (Australia) }\end{array}$ & Human Relations & Artículo & 198 \\
\hline 51 & $\begin{array}{l}\text { Moral awareness in business } \\
\text { organizations: Influences of issue- } \\
\text { related and social context factors } \\
\text { (Butterfield y otros, 2000) }\end{array}$ & $\begin{array}{l}\text { Universidad Estatal de } \\
\text { Washington (Estados } \\
\text { Unidos) }\end{array}$ & $\begin{array}{l}\text { International } \\
\text { Journal of } \\
\text { Accounting } \\
\text { Information } \\
\text { Systems }\end{array}$ & Artículo & 198 \\
\hline 52 & $\begin{array}{l}\text { A quantitative analysis of pricing } \\
\text { behavior in California's wholesale } \\
\text { electricity market during summer } \\
2000 \text { (Joskow y Kahn, 2002) }\end{array}$ & $\begin{array}{l}\text { Analysis Group, Inc. } \\
\text { (Estados Unidos) }\end{array}$ & Energy Journal & Artículo & 197 \\
\hline 53 & $\begin{array}{l}\text { Innovating business models with } \\
\text { co-development partnerships } \\
\text { (Chesbrough y Schwartz, 2007) }\end{array}$ & $\begin{array}{l}\text { Universidad de } \\
\text { California Berkeley } \\
\text { (Estados Unidos) }\end{array}$ & $\begin{array}{l}\text { Research } \\
\text { Technology } \\
\text { Management }\end{array}$ & Artículo & 190 \\
\hline 54 & $\begin{array}{l}\text { Knowledge management technology } \\
\text { and the reproduction of knowledge } \\
\text { work practices (Schultze y Boland Jr, } \\
2000 \text { ) }\end{array}$ & $\begin{array}{l}\text { Escuela de Negocios } \\
\text { Edwin L. Cox (Estados } \\
\text { Unidos) }\end{array}$ & $\begin{array}{l}\text { Journal of Strategic } \\
\text { Information } \\
\text { Systems }\end{array}$ & Artículo & 185 \\
\hline 55 & $\begin{array}{l}\text { What makes process models } \\
\text { understandable? (Mendling y otros, } \\
\text { 2007) }\end{array}$ & $\begin{array}{l}\text { Universidad de } \\
\text { Economía de Viena } \\
\text { (Austria) }\end{array}$ & $\begin{array}{l}\text { Lecture Notes } \\
\text { in Computer } \\
\text { Science (including } \\
\text { subseries Lecture } \\
\text { Notes in Artificial } \\
\text { Intelligence and } \\
\text { Lecture Notes in } \\
\text { Bioinformatics) }\end{array}$ & $\begin{array}{l}\text { Publicación en } \\
\text { congreso }\end{array}$ & 182 \\
\hline 56 & $\begin{array}{l}\text { Time prediction based on process } \\
\text { mining (van der Aalst y otros, 2011) }\end{array}$ & $\begin{array}{l}\text { Universidad de } \\
\text { Eindhoven (Holanda) }\end{array}$ & $\begin{array}{l}\text { Information } \\
\text { Systems }\end{array}$ & Artículo & 174 \\
\hline 57 & $\begin{array}{l}\text { Integrated decision support systems: } \\
\text { A data warehousing perspective } \\
\text { (March y Hevner, 2007) }\end{array}$ & $\begin{array}{l}\text { Schonberger and } \\
\text { Associates Inc. } \\
\text { (Estados Unidos) }\end{array}$ & $\begin{array}{l}\text { Journal of } \\
\text { Operations } \\
\text { Management }\end{array}$ & Artículo & 169 \\
\hline 58 & $\begin{array}{l}\text { Japanese production management: } \\
\text { An evolution-with mixed success } \\
\text { (Schonberger, 2007) }\end{array}$ & $\begin{array}{l}\text { Universidad de } \\
\text { Vanderbilt (Estados } \\
\text { Unidos) }\end{array}$ & $\begin{array}{l}\text { Decision Support } \\
\text { Systems }\end{array}$ & Artículo & 169 \\
\hline 59 & $\begin{array}{l}\text { Is there a liability of foreigness in } \\
\text { global banking? An empirical test of } \\
\text { banks' X-efficiency (Miller y Parkhe, } \\
\text { 2002) }\end{array}$ & $\begin{array}{l}\text { Universidad Estatal } \\
\text { de Michigan (Estados } \\
\text { Unidos) }\end{array}$ & $\begin{array}{l}\text { Strategic } \\
\text { Management } \\
\text { Journal }\end{array}$ & Artículo & 168 \\
\hline
\end{tabular}




\begin{tabular}{|c|c|c|c|c|c|}
\hline No. & Titulo (referencia) & $\begin{array}{l}\text { Afiliación del autor } \\
\text { principal (País) }\end{array}$ & Fuente & $\begin{array}{c}\text { Tipo de } \\
\text { documento }\end{array}$ & Citas \\
\hline 60 & $\begin{array}{l}\text { Towards a multi-objective } \\
\text { optimization approach for improving } \\
\text { energy efficiency in buildings } \\
\text { (Diakaki y otros, 2008) }\end{array}$ & $\begin{array}{l}\text { Instituto Tecnológico } \\
\text { Educativo de Creta } \\
\text { (Grecia) }\end{array}$ & $\begin{array}{l}\text { Energy and } \\
\text { Buildings }\end{array}$ & Artículo & 167 \\
\hline 61 & $\begin{array}{l}\text { A cross-collection mixture model } \\
\text { for comparative text mining (Zhai y } \\
\text { otros, 2004) }\end{array}$ & $\begin{array}{l}\text { Universidad de Illinois } \\
\text { (Estados Unidos) }\end{array}$ & $\begin{array}{l}\text { 10th ACM SIGKDD } \\
\text { International } \\
\text { Conference } \\
\text { on Knowledge } \\
\text { Discovery and Data } \\
\text { Mining }\end{array}$ & $\begin{array}{l}\text { Publicación en } \\
\text { congreso }\end{array}$ & 165 \\
\hline 62 & $\begin{array}{l}\text { Effects of marketing-manufacturing } \\
\text { integration on new product } \\
\text { development time and competitive } \\
\text { advantage (Swink y Song, 2007) }\end{array}$ & INSEAD (Francia) & $\begin{array}{l}\text { Strategic } \\
\text { Management } \\
\text { Journal }\end{array}$ & Artículo & 164 \\
\hline 63 & $\begin{array}{l}\text { When do acquirers earn abnormal } \\
\text { returns? (Capron y Pistre, 2002) }\end{array}$ & $\begin{array}{l}\text { Universidad Estatal } \\
\text { de Michigan (Estados } \\
\text { Unidos) }\end{array}$ & $\begin{array}{l}\text { Journal of } \\
\text { Operations } \\
\text { Management }\end{array}$ & Artículo & 164 \\
\hline 64 & $\begin{array}{l}\text { Towards formal analysis of artifact- } \\
\text { centric business process models } \\
\text { (Bhattacharya y otros, 2007) }\end{array}$ & IBM (Estados Unidos) & $\begin{array}{l}\text { Lecture Notes } \\
\text { in Computer } \\
\text { Science (including } \\
\text { subseries Lecture } \\
\text { Notes in Artificial } \\
\text { Intelligence and } \\
\text { Lecture Notes in } \\
\text { Bioinformatics) }\end{array}$ & $\begin{array}{l}\text { Publicación en } \\
\text { congreso }\end{array}$ & 163 \\
\hline 65 & $\begin{array}{l}\text { Beyond data warehousing: What's } \\
\text { next in business intelligence? } \\
\text { (Golfarelli y otros, 2004) }\end{array}$ & $\begin{array}{l}\text { Universidad de Bolonia } \\
\text { (Italia) }\end{array}$ & $\begin{array}{l}\text { DOLAP: } \\
\text { Proceedings of the } \\
\text { ACM International } \\
\text { Workshop on Data } \\
\text { Warehousing and } \\
\text { OLAP }\end{array}$ & $\begin{array}{l}\text { Publicación en } \\
\text { congreso }\end{array}$ & 160 \\
\hline 66 & $\begin{array}{l}\text { Competition in remanufacturing and } \\
\text { the effects of government subsidies } \\
\text { (Mitra y Webster, 2008) }\end{array}$ & $\begin{array}{l}\text { Tata Consultancy } \\
\text { Services (India) }\end{array}$ & $\begin{array}{l}\text { International } \\
\text { Journal of } \\
\text { Production } \\
\text { Economics }\end{array}$ & Artículo & 159 \\
\hline 67 & $\begin{array}{l}\text { Computer-aided machine-tool } \\
\text { selection based on a Fuzzy-AHP } \\
\text { approach (Durán y Aguilo, 2008) }\end{array}$ & $\begin{array}{l}\text { Universidad Católica de } \\
\text { Valparaíso (Chile) }\end{array}$ & $\begin{array}{l}\text { Expert Systems } \\
\text { with Applications }\end{array}$ & Artículo & 157 \\
\hline 68 & $\begin{array}{l}\text { Review spam detection (Jindal y Liu, } \\
\text { 2007) }\end{array}$ & $\begin{array}{l}\text { Ejército de Estados } \\
\text { Unidos (Estados } \\
\text { Unidos) }\end{array}$ & $\begin{array}{l}\text { Technological } \\
\text { Forecasting and } \\
\text { Social Change }\end{array}$ & Artículo & 156 \\
\hline 69 & $\begin{array}{l}\text { Cluster analysis for data mining and } \\
\text { system identification (Abonyi y Feil, } \\
2007 \text { ) }\end{array}$ & $\begin{array}{l}\text { Universidad de Illinois } \\
\text { (Estados Unidos) }\end{array}$ & $\begin{array}{l}\text { 16th International } \\
\text { World Wide Web } \\
\text { Conference, } \\
\text { WWW2007 }\end{array}$ & $\begin{array}{l}\text { Publicación en } \\
\text { congreso }\end{array}$ & 156 \\
\hline 70 & $\begin{array}{l}\text { Innovation Forecasting (Watts y } \\
\text { Porter, 1997) }\end{array}$ & $\begin{array}{l}\text { Universidad de Panonia } \\
\text { (Hungría) }\end{array}$ & $\begin{array}{l}\text { Cluster Analysis } \\
\text { for Data Mining } \\
\text { and System } \\
\text { Identification } \\
\end{array}$ & Libro & 156 \\
\hline 71 & $\begin{array}{l}\text { Business-to-business adoption of } \\
\text { eCommerce in China (Tan y otros, } \\
\text { 2007) }\end{array}$ & $\begin{array}{l}\text { Universidad Atlántica } \\
\text { de Florida (Estados } \\
\text { Unidos) }\end{array}$ & $\begin{array}{l}\text { Journal of } \\
\text { Operations } \\
\text { Management }\end{array}$ & Artículo & 154 \\
\hline 72 & $\begin{array}{l}\text { Integrated product development } \\
\text { practices and competitive capabilities: } \\
\text { The effects of uncertainty, } \\
\text { equivocality, and platform strategy } \\
\text { (Koufteros y otros, 2002) }\end{array}$ & $\begin{array}{l}\text { Universidad de } \\
\text { Westminster (Reino } \\
\text { Unido) }\end{array}$ & $\begin{array}{l}\text { Information and } \\
\text { Management }\end{array}$ & Artículo & 154 \\
\hline 73 & $\begin{array}{l}\text { Practical Text Mining and Statistical } \\
\text { Analysis for Non-structured Text } \\
\text { Data Applications (Miner y otros, } \\
\text { 2012) }\end{array}$ & $\begin{array}{l}\text { Universidad de } \\
\text { Minnesota (Estados } \\
\text { Unidos) }\end{array}$ & $\begin{array}{l}\text { Practical Text } \\
\text { Mining and } \\
\text { Statistical Analysis } \\
\text { for Non-structured } \\
\text { Text Data } \\
\text { Applications }\end{array}$ & Libro & 152 \\
\hline
\end{tabular}




\begin{tabular}{|c|c|c|c|c|c|}
\hline No. & Titulo (referencia) & $\begin{array}{l}\text { Afiliación del autor } \\
\text { principal (País) }\end{array}$ & Fuente & $\begin{array}{c}\text { Tipo de } \\
\text { documento }\end{array}$ & Citas \\
\hline 74 & $\begin{array}{l}\text { Critical success factors for business } \\
\text { intelligence systems (Yeoh y } \\
\text { Koronios, 2010) }\end{array}$ & $\begin{array}{l}\text { Universidad del Sur de } \\
\text { Australia (Australia) }\end{array}$ & $\begin{array}{l}\text { Journal of } \\
\text { Computer } \\
\text { Information } \\
\text { Systems } \\
\end{array}$ & Artículo & 152 \\
\hline 75 & $\begin{array}{l}\text { Discovery of web robot sessions } \\
\text { based on their navigational patterns } \\
\text { (Tan y Kumar, 2002) }\end{array}$ & $\begin{array}{l}\text { Universidad Estatal } \\
\text { de Michigan (Estados } \\
\text { Unidos) }\end{array}$ & $\begin{array}{l}\text { Data Mining } \\
\text { and Knowledge } \\
\text { Discovery }\end{array}$ & Artículo & 152 \\
\hline 76 & $\begin{array}{l}\text { Machine learning and data mining } \\
\text { (Kononenko y Kukar, 2007) }\end{array}$ & $\begin{array}{l}\text { Universidad de } \\
\text { Liubliana (Eslovenia) }\end{array}$ & $\begin{array}{l}\text { Machine Learning } \\
\text { and Data Mining }\end{array}$ & Libro & 150 \\
\hline 77 & $\begin{array}{l}\text { Green manufacturing: An evaluation } \\
\text { of environmentally sustainable } \\
\text { manufacturing practices and their } \\
\text { impact on competitive outcomes } \\
\text { (Rusinko, 2007) }\end{array}$ & $\begin{array}{l}\text { Universidad de } \\
\text { Filadelfia (Estados } \\
\text { Unidos) }\end{array}$ & $\begin{array}{l}\text { IEEE Transactions } \\
\text { on Engineering } \\
\text { Management }\end{array}$ & Artículo & 146 \\
\hline 78 & $\begin{array}{l}\text { The dynamic structure of } \\
\text { management support systems: } \\
\text { Theory development, research focus, } \\
\text { and direction (Clark y otros, 2007) }\end{array}$ & $\begin{array}{l}\text { Politécnico de Milano } \\
\text { (Italia) }\end{array}$ & $\begin{array}{l}\text { Strategic } \\
\text { Management } \\
\text { Journal }\end{array}$ & Revisión & 144 \\
\hline 79 & $\begin{array}{l}\text { Alliance form: A test of the } \\
\text { contractual and competence } \\
\text { perspectives (Colombo, 2003) }\end{array}$ & $\begin{array}{l}\text { Universidad Estatal } \\
\text { de Luisiana (Estados } \\
\text { Unidos) }\end{array}$ & $\begin{array}{l}\text { MIS Quarterly: } \\
\text { Management } \\
\text { Information } \\
\text { Systems }\end{array}$ & Artículo & 144 \\
\hline 80 & $\begin{array}{l}\text { The integration of business } \\
\text { intelligence and knowledge } \\
\text { management (Cody y otros, 2002) }\end{array}$ & IBM (Estados Unidos) & $\begin{array}{l}\text { IBM Systems } \\
\text { Journal }\end{array}$ & Artículo & 143 \\
\hline 81 & $\begin{array}{l}\text { Six Sigma literature: A review and } \\
\text { agenda for future research (Brady y } \\
\text { Allen, 2006) }\end{array}$ & $\begin{array}{l}\text { LaBarge, Inc. (Estados } \\
\text { Unidos) }\end{array}$ & $\begin{array}{l}\text { Information and } \\
\text { Management }\end{array}$ & Artículo & 140 \\
\hline 82 & $\begin{array}{l}\text { An exploratory examination of } \\
\text { the relationship between flexible } \\
\text { IT infrastructure and competitive } \\
\text { advantage (Byrd y Turner, 2001) }\end{array}$ & $\begin{array}{l}\text { Universidad de Auburn } \\
\text { (Estados Unidos) }\end{array}$ & $\begin{array}{l}\text { Quality and } \\
\text { Reliability } \\
\text { Engineering } \\
\text { International }\end{array}$ & Revisión & 140 \\
\hline 83 & $\begin{array}{l}\text { Innovativeness and organizational } \\
\text { innovation in total quality oriented } \\
\text { firms: The moderating role of } \\
\text { market turbulence (Santos-Vijande y } \\
\text { Álvarez-González, 2007) }\end{array}$ & $\begin{array}{l}\text { Escuela de Negocios } \\
\text { Goizueta (Estados } \\
\text { Unidos) }\end{array}$ & Technovation & Artículo & 139 \\
\hline 84 & $\begin{array}{l}\text { Turnover of information technology } \\
\text { workers: Examining empirically } \\
\text { the influence of attitudes, job } \\
\text { characteristics, and external markets } \\
\text { (Thatcher y otros, 2002) }\end{array}$ & $\begin{array}{l}\text { Universidad de } \\
\text { Clemson (Estados } \\
\text { Unidos) }\end{array}$ & $\begin{array}{l}\text { Management } \\
\text { Science }\end{array}$ & Artículo & 139 \\
\hline 85 & $\begin{array}{l}\text { Strategic factor market intelligence: } \\
\text { An application of information } \\
\text { economics to strategy formulation } \\
\text { and competitor intelligence (Makadok } \\
\text { y Barney, 2001) }\end{array}$ & $\begin{array}{l}\text { Universidad de Oviedo } \\
\text { (España) }\end{array}$ & $\begin{array}{l}\text { Journal of } \\
\text { Management } \\
\text { Information } \\
\text { Systems }\end{array}$ & Artículo & 139 \\
\hline 86 & $\begin{array}{l}\text { Business intelligence in Blogs: } \\
\text { Understanding consumer interactions } \\
\text { and communities (Chau y Xu, 2012) }\end{array}$ & $\begin{array}{l}\text { Universidad de Auburn } \\
\text { (China) }\end{array}$ & $\begin{array}{l}\text { MIS Quarterly: } \\
\text { Management } \\
\text { Information } \\
\text { Systems } \\
\end{array}$ & Artículo & 138 \\
\hline 87 & $\begin{array}{l}\text { Business intelligence: An analysis of } \\
\text { the literature (Jourdan y otros, 2008) }\end{array}$ & $\begin{array}{l}\text { Universidad de Hong } \\
\text { Kong (China) }\end{array}$ & $\begin{array}{l}\text { Information } \\
\text { Systems } \\
\text { Management }\end{array}$ & Artículo & 138 \\
\hline 88 & $\begin{array}{l}\text { Business Intelligence: Data Mining } \\
\text { and Optimization for Decision Making } \\
\text { (Vercellis, 2009) }\end{array}$ & $\begin{array}{l}\text { Politécnico de Milano } \\
\text { (Italia) }\end{array}$ & $\begin{array}{l}\text { Business } \\
\text { Intelligence: } \\
\text { Data Mining and } \\
\text { Optimization for } \\
\text { Decision Making }\end{array}$ & Libro & 136 \\
\hline
\end{tabular}




\begin{tabular}{|c|c|c|c|c|c|}
\hline No. & Titulo (referencia) & $\begin{array}{l}\text { Afiliación del autor } \\
\text { principal (País) }\end{array}$ & Fuente & $\begin{array}{c}\text { Tipo de } \\
\text { documento }\end{array}$ & Citas \\
\hline 89 & $\begin{array}{l}\text { Sources of volume flexibility and } \\
\text { their impact on performance (Jack y } \\
\text { Raturi, 2002) }\end{array}$ & $\begin{array}{l}\text { Universidad de } \\
\text { Alabama en } \\
\text { Birmingham (Estados } \\
\text { Unidos) } \\
\end{array}$ & $\begin{array}{l}\text { Journal of } \\
\text { Operations } \\
\text { Management }\end{array}$ & Artículo & 136 \\
\hline 90 & $\begin{array}{l}\text { Risk measures and comonotonicity: } \\
\text { A review (Dhaene y otros, 2006) }\end{array}$ & $\begin{array}{l}\text { Universidad de } \\
\text { Ámsterdam (Países } \\
\text { Bajos) }\end{array}$ & $\begin{array}{l}\text { 12th International } \\
\text { Conference on } \\
\text { World Wide Web, } \\
\text { WWW } 2003\end{array}$ & $\begin{array}{l}\text { Publicación en } \\
\text { congreso }\end{array}$ & 135 \\
\hline 91 & $\begin{array}{l}\text { p-Queries: Enabling querying } \\
\text { for semantic associations on the } \\
\text { semantic web (Anyanwu y Sheth, } \\
2003 \text { ) }\end{array}$ & $\begin{array}{l}\text { Universidad de Georgia } \\
\text { (Estados Unidos) }\end{array}$ & $\begin{array}{l}\text { Management } \\
\text { Science }\end{array}$ & Artículo & 135 \\
\hline 92 & $\begin{array}{l}\text { Breaking through the clutter: } \\
\text { Benefits of advertisement originality } \\
\text { and familiarity for brand attention } \\
\text { and memory (Pieters y otros, 2002) }\end{array}$ & $\begin{array}{l}\text { Universidad de Tilburg } \\
\text { (Holanda) }\end{array}$ & Stochastic Models & $\begin{array}{l}\text { Publicación en } \\
\text { congreso }\end{array}$ & 135 \\
\hline 93 & $\begin{array}{l}\text { Innovative competence, exploration } \\
\text { and exploitation: The influence } \\
\text { of technological diversification } \\
\text { (Quintana-García y Benavides- } \\
\text { Velasco, 2008) }\end{array}$ & $\begin{array}{l}\text { Universidad de Málaga } \\
\text { (España) }\end{array}$ & Research Policy & Artículo & 134 \\
\hline 94 & $\begin{array}{l}\text { Predicting online-purchasing } \\
\text { behaviour (Van Den Poel y Buckinx, } \\
2005 \text { ) }\end{array}$ & $\begin{array}{l}\text { AAG Associates } \\
\text { (Belgica) }\end{array}$ & $\begin{array}{l}\text { European Journal } \\
\text { of Operational } \\
\text { Research }\end{array}$ & Artículo & 132 \\
\hline 95 & $\begin{array}{l}\text { Customers' choice among retail } \\
\text { energy suppliers: The willingness- } \\
\text { to-pay for service attributes (Goett y } \\
\text { otros, 2000) }\end{array}$ & $\begin{array}{l}\text { Universidad de Ghent } \\
\text { (Estados Unidos) }\end{array}$ & Energy Journal & Artículo & 132 \\
\hline 96 & $\begin{array}{l}\text { Business intelligence for enterprise } \\
\text { systems: A survey (Duan y Xu, } \\
2012 \text { ) }\end{array}$ & $\begin{array}{l}\text { Instituto Tecnológico } \\
\text { de New Jersey } \\
\text { (Estados Unidos) }\end{array}$ & $\begin{array}{l}\text { IEEE Transactions } \\
\text { on Industrial } \\
\text { Informatics } \\
\end{array}$ & Revisión & 131 \\
\hline 97 & $\begin{array}{l}\text { Management support with structured } \\
\text { and unstructured data - An } \\
\text { integrated business intelligence } \\
\text { framework (Baars y Kemper, 2008) }\end{array}$ & $\begin{array}{l}\text { Universidad de la } \\
\text { Ciudad de New York } \\
\text { (Estados Unidos) }\end{array}$ & $\begin{array}{l}\text { Strategic } \\
\text { Management } \\
\text { Journal }\end{array}$ & Artículo & 129 \\
\hline 98 & $\begin{array}{l}\text { The measurement of business } \\
\text { intelligence (Lönnqvist y Pirttimäki, } \\
\text { 2006) }\end{array}$ & $\begin{array}{l}\text { Universidad de } \\
\text { Stuttgart (Alemania) }\end{array}$ & $\begin{array}{l}\text { Information } \\
\text { Systems } \\
\text { Management } \\
\end{array}$ & Artículo & 129 \\
\hline 99 & $\begin{array}{l}\text { Liability of foreigness in global } \\
\text { competition? Financial service } \\
\text { affiliates in the city of London } \\
\text { (Nachum, 2003) }\end{array}$ & $\begin{array}{l}\text { Universidad } \\
\text { Tecnológica de } \\
\text { Tampere (Finlandia) }\end{array}$ & $\begin{array}{l}\text { Information } \\
\text { Systems } \\
\text { Management }\end{array}$ & Revisión & 129 \\
\hline 100 & $\begin{array}{l}\text { Mining comparative opinions from } \\
\text { customer reviews for Competitive } \\
\text { Intelligence (Xu y otros, 2011) }\end{array}$ & $\begin{array}{l}\text { Universidad de la } \\
\text { Ciudad de Hong Kong } \\
\text { (China) }\end{array}$ & $\begin{array}{l}\text { Decision Support } \\
\text { Systems }\end{array}$ & Artículo & 126 \\
\hline 101 & $\begin{array}{l}\text { Big Data, Big Analytics: Emerging } \\
\text { Business Intelligence and Analytic } \\
\text { Trends for Today's Businesses (Minelli } \\
\text { y otros, 2013) }\end{array}$ & $\begin{array}{l}\text { Mu Sigma (Estados } \\
\text { Unidos) }\end{array}$ & $\begin{array}{l}\text { Big Data, Big } \\
\text { Analytics: } \\
\text { Emerging Business } \\
\text { Intelligence and } \\
\text { Analytic Trends for } \\
\text { Today's Businesses } \\
\end{array}$ & Libro & 125 \\
\hline 102 & $\begin{array}{l}\text { Performance of global new product } \\
\text { development programs: A resource- } \\
\text { based view (Kleinschmidt y otros, } \\
2007 \text { ) }\end{array}$ & $\begin{array}{l}\text { Universidad McMaster } \\
\text { (Estados Unidos) }\end{array}$ & $\begin{array}{l}\text { Journal of Product } \\
\text { Innovation } \\
\text { Management }\end{array}$ & Artículo & 125 \\
\hline 103 & $\begin{array}{l}\text { Challenging the interoperability } \\
\text { between computers in industry with } \\
\text { MDA and SOA (Jardim-Goncalves y } \\
\text { otros, 2006) }\end{array}$ & $\begin{array}{l}\text { Universidad Nueva de } \\
\text { Lisboa (Portugal) }\end{array}$ & $\begin{array}{l}\text { Computers in } \\
\text { Industry }\end{array}$ & Artículo & 123 \\
\hline 104 & $\begin{array}{l}\text { A theory of purchasing's contribution } \\
\text { to business performance (González- } \\
\text { Benito, 2007) }\end{array}$ & $\begin{array}{l}\text { Universidad de } \\
\text { Salamanca (España) }\end{array}$ & $\begin{array}{l}\text { Journal of } \\
\text { Operations } \\
\text { Management } \\
\end{array}$ & Artículo & 122 \\
\hline 105 & $\begin{array}{l}\text { Industrial symbiosis in China: A case } \\
\text { study of the Guitang Group (Zhu y } \\
\text { otros, 2007) }\end{array}$ & $\begin{array}{l}\text { Universidad de Dalian } \\
\text { (China) }\end{array}$ & $\begin{array}{l}\text { Journal of } \\
\text { Industrial Ecology }\end{array}$ & Revisión & 121 \\
\hline
\end{tabular}




\begin{tabular}{|c|c|c|c|c|c|}
\hline No. & Titulo (referencia) & $\begin{array}{l}\text { Afiliación del autor } \\
\text { principal (País) }\end{array}$ & Fuente & $\begin{array}{c}\text { Tipo de } \\
\text { documento }\end{array}$ & Citas \\
\hline 106 & $\begin{array}{l}\text { From BPMN process models to BPEL } \\
\text { Web services (Ouyang y otros, 2006) }\end{array}$ & $\begin{array}{l}\text { Universidad } \\
\text { Tecnológica de } \\
\text { Queensland (Australia) }\end{array}$ & $\begin{array}{l}\text { ICWS 2006: } 2006 \\
\text { IEEE International } \\
\text { Conference on Web } \\
\text { Services }\end{array}$ & $\begin{array}{l}\text { Publicación en } \\
\text { congreso }\end{array}$ & 120 \\
\hline 107 & $\begin{array}{l}\text { IT capabilities, process-oriented } \\
\text { dynamic capabilities, and firm } \\
\text { financial performance (Kim y otros, } \\
\text { 2011) }\end{array}$ & $\begin{array}{l}\text { Universidad de } \\
\text { Konyang (Corea del } \\
\text { Sur) }\end{array}$ & $\begin{array}{l}\text { Journal of the } \\
\text { Association of } \\
\text { Information } \\
\text { Systems }\end{array}$ & Artículo & 118 \\
\hline 108 & $\begin{array}{l}\text { A visual framework for knowledge } \\
\text { discovery on the web: An empirical } \\
\text { study of business intelligence } \\
\text { exploration (Chung y otros, 2005) } \\
\end{array}$ & $\begin{array}{l}\text { Universidad de Texas } \\
\text { (Estados Unidos) }\end{array}$ & \begin{tabular}{|l|} 
Journal of \\
Management \\
Information \\
Systems \\
\end{tabular} & Artículo & 117 \\
\hline 109 & $\begin{array}{l}\text { Show me the money!: Deriving the } \\
\text { pricing power of product features by } \\
\text { mining consumer reviews (Archak y } \\
\text { otros, 2007) }\end{array}$ & $\begin{array}{l}\text { Escuela de Negocios } \\
\text { Leonard N. Stern de la } \\
\text { Universidad de Nueva } \\
\text { York (Estados Unidos) }\end{array}$ & \begin{tabular}{|l|} 
ACM SIGKDD \\
International \\
Conference \\
on Knowledge \\
Discovery and Data \\
Mining \\
\end{tabular} & $\begin{array}{l}\text { Publicación en } \\
\text { congreso }\end{array}$ & 116 \\
\hline 110 & $\begin{array}{l}\text { Customer churn analysis: Churn } \\
\text { determinants and mediation effects } \\
\text { of partial defection in the Korean } \\
\text { mobile telecommunications service } \\
\text { industry (Ahn y otros, 2006) }\end{array}$ & $\begin{array}{l}\text { Instituto Avanzado de } \\
\text { Ciencia y Tecnología de } \\
\text { Corea (Corea del Sur) }\end{array}$ & $\begin{array}{l}\text { Telecommunica- } \\
\text { tions Policy }\end{array}$ & Artículo & 116 \\
\hline 111 & $\begin{array}{l}\text { Developing capabilities and } \\
\text { competence for sustainable business } \\
\text { management as innovation: a } \\
\text { research agenda (van Kleef y } \\
\text { Roome, 2007) }\end{array}$ & $\begin{array}{l}\text { Trees Inventing } \\
\text { (Holanda) }\end{array}$ & $\begin{array}{l}\text { Journal of Cleaner } \\
\text { Production }\end{array}$ & Artículo & 115 \\
\hline 112 & $\begin{array}{l}\text { Knowledge management for } \\
\text { sustainable competitiveness in small } \\
\text { and medium surveying practices } \\
\text { (Egbu y otros, 2005) }\end{array}$ & $\begin{array}{l}\text { Universidad de } \\
\text { Glasgow Caledonian } \\
\text { (Reino Unido) }\end{array}$ & $\begin{array}{l}\text { Information } \\
\text { Systems Research }\end{array}$ & Artículo & 113 \\
\hline 113 & $\begin{array}{l}\text { The net-enabled business innovation } \\
\text { cycle and the evolution of dynamic } \\
\text { capabilities (Zahra y George, 2002) }\end{array}$ & $\begin{array}{l}\text { Universidad Estatal } \\
\text { de Georgia (Estados } \\
\text { Unidos) }\end{array}$ & Structural Survey & Artículo & 113 \\
\hline
\end{tabular}




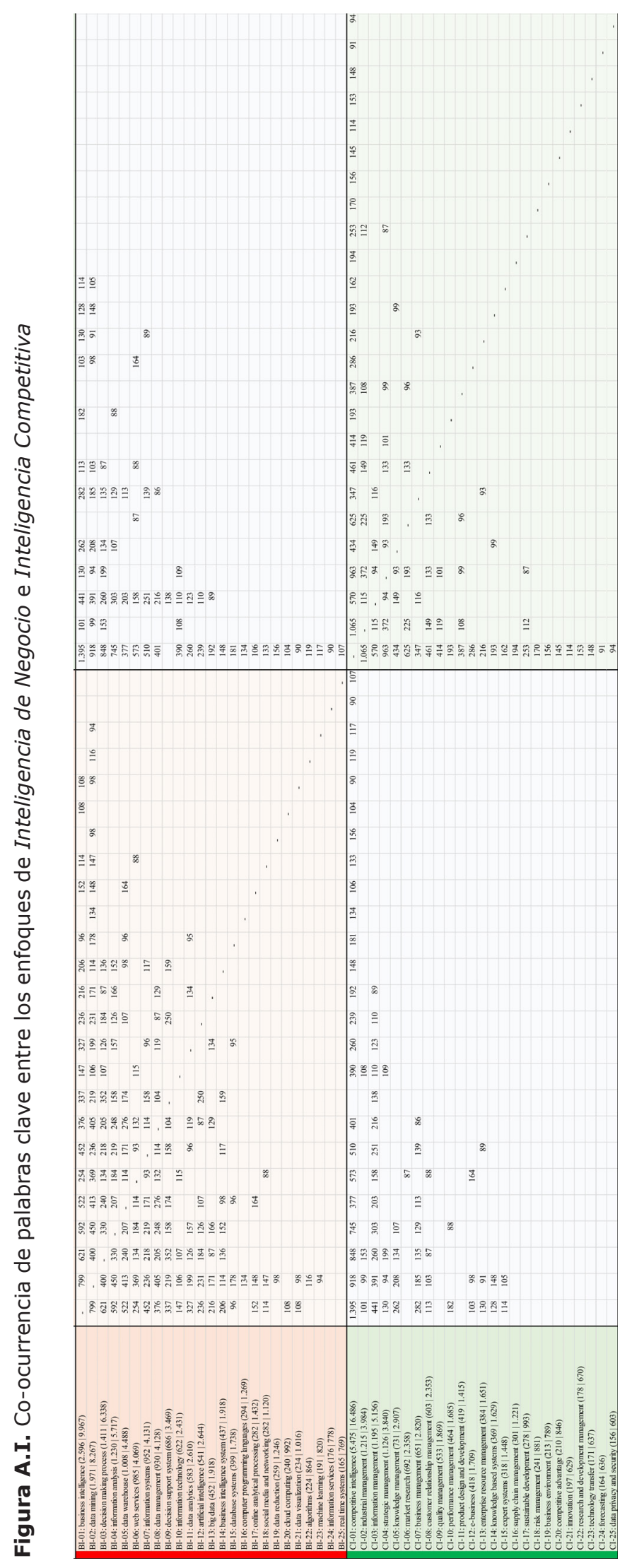

